\title{
Transient Landing Dynamics Analysis for A Lunar Lander with Random and Interval fields
}

\author{
Zhao-Yue Chen ${ }^{\mathrm{a}, *}$, Maurice Imholz ${ }^{\mathrm{b}}$, Liu Li ${ }^{\mathrm{a}, \mathrm{c}}$, Matthias Faes $^{\mathrm{b}}$, David \\ Moens $^{\mathrm{b}}$ \\ ${ }^{a}$ School of Aerospace Engineering, Beijing Institute of Technology, Zhongguancun South \\ Street 5, 100081 Beijing, China \\ ${ }^{b}$ KU Leuven - Department of Mechanical Engineering, Jan De Nayerlaan 5, 2860 \\ St.-Katelijne-Waver, Belgium \\ ${ }^{c}$ Key Laboratory of Dynamics and Control of Flight Vehicle, Ministry of Education, \\ 100081 Beijing, China
}

\begin{abstract}
This paper presents an objective comparison of random fields and interval fields to propagate spatial uncertainty, based on a finite element model of a lunar lander. The impulse based substructuring method is used to improve the analysis efficiency. The spatially uncertain input parameters are modeled by both random fields and interval fields. The objective of this work is to compare the applicability of both approaches in an early design stage under scarce information regarding the occurring spatial parameter variability. Focus is on the definition of the input side of the problem under this scarce knowledge, as well as the interpretation of the analysis outcome. To obtain an objective comparison between both approaches, the gradients in the interval field are tuned towards the gradients present in the random field. The result shows a very similar dependence and correlation structure between the local properties for both approaches. Furthermore, through the transient dynamic estimation, it is shown that the response ranges that are predicted by the interval field and random field are very close to each
\end{abstract}


other.

Keywords: random field, interval field, uncertainty quantification, impulse based substructuring, lunar lander

\section{Introduction}

Numerical modeling techniques have gained increasing interest in engineering, and the deterministic Finite Element (FE) method has become an indispensable tool for an engineer in various design stages. However, it is often impossible to determine all numerical model parameters deterministically, as the true parameter value is uncertain or inherently variable. Consequently, deterministic numerical analyses of the model are inadequate when a reliable and robust design is pursued [1]. By applying non-deterministic approaches for FE simulations throughout different stages of a product design, a more robust and reliable design can be achieved, while reducing the cost of the experimental test phase. As such, several non-deterministic finite element methods have been studied $[1,2,3]$.

Two distinctive philosophies exist for propagating non-deterministic quantities through FE analyses, namely the probabilistic and the possibilistic approach. In a probabilistic approach, uncertainty is represented as a probability density function (PDF) and thus propagated through an FE model using sampling or perturbation methods, possibly through a meta-model [4]. Possibilistic approaches on the other hand include interval methods [1], fuzzy approaches [5] or techniques coming from the field of imprecise probabilities [3]. A large body of literature has been dedicated to the comparison

\footnotetext{
${ }^{*}$ Corresponding author

Email address: chenzhaoyue2909@sina.com (Zhao-Yue Chen) Preprint submitted to Applied Mathematical Modelling
} 
of both philosophies in a forward [5] and inverse uncertainty quantification setting [6]. These studies show that, instead of competing, both approaches are complementary in a design context, and in fact the selection of the most appropriate approach should be based on the quantity and quality of information that is available to the analyst [7]. The probabilistic approach has reached wide acceptance among academia and industry and it has been validated in a wide variety of applications ranging from machine design to bio-medics $[8,9,10,11,12]$. However, in engineering applications, this approach often proves to be inconvenient, mainly due to the large amount of data necessary for the identification of a PDF or the corresponding statistical moments. Therefore, in practice, many researchers assume the data to follow a Gaussian distribution. This possibly results in a severe misjudgment of the non-deterministic data structure and correspondingly in an unrealistic assessment of the uncertainty in the analysis results [1].

Also for the modeling of spatial uncertainty, both probabilistic and possibilistic methods have been introduced. In a probabilistic context, a random field is applied to represent a random quantity at each point of a continuous domain. For computational purposes, the random field has to be expressed using a finite number of random variables. This step is referred to as random field discretization. Various methods for random field discretization have been published in literature. A comprehensive overview is given in [13].

Interval fields on the other hand were introduced only recently. In 2011, Moens et al. [14] proposed an interval field model which utilizes only upper and lower bounds for the representation of the variability of a spatial parameter. In the interval field model, an uncertain but bounded spatial 
parameter is represented as a superposition of a series of base vectors using interval factors, through which the spatial dependency of the parameter can be considered [15]. Afterwards, Muscolino [16] extended this concept and proposed another interval field model based on the improved interval analysis via the concept of the extra unitary interval. Subsequently, this interval field model was applied to static analysis of beams with uncertain Young's modulus under deterministic static loads. Recently, Wu and Gao [17] carried out the static plane stress analysis of continuous structures involving interval fields, by which the upper and lower bounds of structural responses can be determined.

Some comparisons between interval and random fields, as well as hybrid approaches have been reported recently. Sofi [18] analyzed response variability of Euler-Bernoulli beams with spatially varying uncertainties using both a probabilistic and non-probabilistic framework. Yin [19] analyzed the response of structural-acoustic systems with random and interval fields. Some more recent approaches are combining interval fields with random fields. Gao and $\mathrm{Wu}[20,21]$ investigated uncertain static analysis and reliability assessment of engineering structures with hybrid stochastic and non-stochastic uncertain parameters. Feng et al. [22] also present a robust non-deterministic free vibration analysis for engineering structures involving hybrid random and interval uncertain system parameters. Faes et al. [23] applied a modeling approach consisting of a combination of interval and random fields, and studied the application of this approach on the resulting polymorphic uncertainty in the mechanical performance of the PA-12 parts produced via laser sintering.

However, despite this available literature, an important open question 
remains: "given only limited information on the variability levels of a field parameter, which approach provides the most informative results, and how do the results obtained by random fields and interval fields that were fitted to the best estimate of the analyst compare?". This question is of paramount importance in engineering practice when selecting an approach to grasp the spatial uncertainty present in the analysis under consideration. For example, a designer who is faced with heterogeneous material properties with substantial uncertainty levels due to manufacturing variability, but at the same time, only has limited data, needs an answer to this question. Note that in this specific situation, knowledge about the sensitivity of the design performance to this variability might already be very beneficial for design decisions.

Therefore, in this paper, we conduct a thorough comparison of random field and interval field analysis, where utmost care is taken to define equivalent problems at the input side of the problem. First, we discuss the main aspects of the random field and interval field finite element methods and their application for spatial uncertainty modeling. In this context, we specifically tune the gradients of the realisations of the interval field to those seen in the random field, as to allow for direct comparison. Then, we compare both modelling theories on a realistic case, namely the transient dynamic analysis of a lunar lander. A simplified finite element model of the lunar lander is built. Transient dynamic analysis is performed based on the Impulse Based Substructuring (IBS) approach [24]. The uncertain input parameters are modeled and propagated by both random fields and interval fields. Finally, we compare the transient dynamic response estimated by both approaches. The main contributions of this paper with respect to 
the state-of-the-art are as such (1) an approach to construct an interval field with an autodependence that closely resembles the autocorrelation observed in a random field and (2) new insights in the comparison of both field modelling approaches.

The paper is structured as follows. In section 1, a brief introduction of uncertainty methods is presented. Section 2 and 3 give a concise introduction to random field and interval field methods. In section 4, the numerical model of the lunar lander is presented and the deterministic dynamic analysis of the landing procedure is discussed. Section 5 continues with the definition of the uncertain input parameters based on the random field and interval field approaches and a comparison between both methods is performed. In section 6, the input parameters are propagated to dynamic analysis results and the comparison of interval and random field is conducted. Section 7 finally summarizes the main conclusions.

\section{Random field finite element method}

\subsection{Random field theory}

Uncertain input parameters with inherent spatial variability are usually modeled by means of random fields. A random field represents a random quantity over a continuous domain, and hence, consists of an infinite number of correlated random variables. A continuous random field $H(r, \theta)$ is defined as a random function that describes a random quantity at each point $r \in \Omega$ of a continuous domain $\Omega \subset R^{d}, d \in \mathbb{N}_{>0}$, where $\theta \in \Theta$ is a coordinate in the sample space $\Theta$, and $(\Theta, F, P)$ is a complete probability space with $\mathrm{F}$ a set of events and $\mathrm{P}$ the assignment of probabilities to the events. If the random quantity attached to each point $r$ is a random variable, the random 
field is said to be uni-variate or real-valued. If the random quantity is a random vector, the field is called multivariate. The dimension $d$ of a random field is the dimension of its topological space $\Omega$. One usually distinguishes between a one- and a multi-dimensional random field.

The field is said to be Gaussian if the distribution of $\left(H\left(r_{1}, \theta\right), \ldots H\left(r_{n}, \theta\right)\right)$ is jointly Gaussian for any $\left(r_{1}, \ldots r_{n}\right) \in \Omega$ and any $n \in \mathbb{N}_{>0}$. A Gaussian field is completely defined by its mean function $\mu \in \Omega \mapsto \mathbb{R}$ and autocovariance function $\operatorname{Cov}: \Omega \times \Omega \mapsto \mathbb{R}$. The auto-covariance function can be expressed as $\operatorname{Cov}\left(r, r^{\prime}\right)=\sigma(r) \cdot \sigma\left(r^{\prime}\right) \cdot \rho\left(r, r^{\prime}\right)$, where $\sigma \in \Omega \mapsto \mathbb{R}$ is the standard deviation function of the random field and $\rho: \Omega \times \Omega \mapsto[-1,1]$ is its auto-correlation function.

For computational purposes, the random field has to be approximated using a finite number of random variables. The approximation $\hat{H}(\cdot)$ of a continuous random field $H(\cdot)$ by a finite set of random variables is referred to as random field discretization. Betz et al. [13] give a comprehensive overview of random field discretization methods.

\subsection{The Karhunen-Loève expansion}

The Karhunen-Loève (KL) expansion is one of the random field discretization methods which approximate the random field by a finite sum of products of deterministic spatial functions and random variables. The KL expansion was introduced in the engineering community by Spanos and Ghanem [25]. The expansion requires the solution of a Fredholm integral eigenvalue problem (IEVP), whose integral kernel is the auto-covariance function of the field. Analytical solutions of the IEVP can be obtained only for specific types of auto-covariance functions defined on rectangular do- 
mains. For random fields with arbitrary auto-covariance functions defined on domains of complex geometrical shape, the solution of the IEVP needs to be approximated numerically.

The Karhunen-Loève expansion is a series expansion method for the representation of a random field. The expansion is based on a spectral decomposition of the auto-covariance function of the field. It states that a second-order random field can be represented exactly by the following expansion:

$$
H(r, \theta)=\mu(r)+\sum_{i=1}^{\infty} \sqrt{\lambda_{i}} \varphi_{i}(r) \xi_{i}(\theta)
$$

where $\mu(r)$ is the mean function of the field, $\xi_{i}(\theta): \Theta \mapsto \mathbb{R}$ are standard uncorrelated random variables and $\lambda_{i} \in[0,+\infty), \varphi_{i}: \Omega \mapsto \mathbb{R}$ are the eigenvalues and eigenfunctions of the auto-covariance kernel obtained from solving the homogeneous Fredholm integral equation of the second kind:

$$
\int_{\Omega} \operatorname{Cov}\left(r, r^{\prime}\right) \varphi_{i}\left(r^{\prime}\right) d r^{\prime}=\lambda_{i} \varphi_{i}(r)
$$

In this context, the auto-covariance function $\operatorname{Cov}\left(r, r^{\prime}\right)$ is also referred to as kernel function. Any valid covariance function is a bounded, symmetric and positive semi-definite kernel. Moreover, a continuous kernel function is assumed. Note that the kernel does not have to be stationary. According to Mercer's theorem, the eigenvalues $\lambda_{i}$ are non negative, the eigenfunctions corresponding to positive eigenvalues are continuous and orthogonal to each other, and the kernel function can be written as the uniformly convergent expansion:

$$
\operatorname{Cov}\left(r, r^{\prime}\right)=\sum_{\mathrm{i}=1}^{\infty} \lambda_{i} \varphi_{i}(r) \varphi_{i}\left(r^{\prime}\right)
$$


where the eigenfunctions in the expression are normalized. Consequently, the eigenfunctions are orthonormal with respect to each other, i.e.:

$$
\int_{\Omega} \varphi_{i}(r) \varphi_{j}(r) d r=\delta_{i j}
$$

where $\delta_{i j}$ is the Kronecker delta. As such, they form a complete basis of the space $L^{2}(\Omega)$ of square integrable functions $\Omega$.

If the random field $H(r, \theta)$ is Gaussian, then $\xi_{i}(\theta)$ are independent standard normal random variables. In any other case, the joint distribution of $\xi_{i}(\theta)$ is more involved to obtain. Hence, the KL expansion is mainly applicable to the discretization of Gaussian fields.

The direct modeling of non-Gaussian random fields by means of the KL expansion was discussed by Phoon [26]. In this work, the authors proposed an iterative framework to simulate non-stationary non-Gaussian processes. The procedure was refined for highly skewed non-Gaussian processes by Phoon et al. in [27]. Moreover, non-Gaussian fields are commonly modeled by combining the KL expansion with the polynomial chaos expansion. Ghanem [28] proposed a general framework in which the non-Gaussian field is projected onto an orthogonal polynomial basis with argument an underlying Gaussian field that is then discretized by the KL expansion. Matthies and Keese [29] proposed to perform the KL expansion of the non-Gaussian field and project the random variables involved in the expansion to an underlying independent Gaussian random variable space. Grigoriu [30] developed a simulation algorithm for generating realizations of non-Gaussian stationary translation processes with a specified marginal distribution and covariance function. 
Since it is in practice impossible to compute with an infinite number of random variables, the KL expansion is usually truncated after M terms. The random field approximation of the truncated KL expansion given in Eq. 1 can be written as:

$$
H(r, \theta) \approx \mu(r)+\sum_{i=1}^{M} \sqrt{\lambda_{i}} \varphi_{i}(r) \xi_{i}(\theta)
$$

\subsection{Galerkin method to solve the KL expansion}

Integral eigenvalue problems of the type given in Eq. 2 are difficult to solve analytically except for a few auto-covariance functions defined on domains $\Omega$ of simple geometric shape. Analytical solutions for exponential and triangular kernels are discussed for one-dimensional domains in [31]. Extensions to multi-dimensional rectangular domains can be derived assuming a separable covariance structure. In practice, the integral eigenvalue problem is usually solved numerically.

Numerical algorithms for the solution of the Fredholm integral eigenvalue problems approximate the eigenfunctions by a set of functions $h_{j}$ : $\Omega \mapsto \mathbb{R}$ as:

$$
\varphi_{i}(r) \approx \hat{\varphi}_{i}(r)=\sum_{i=1}^{N} d_{j}^{i} h_{j}
$$

where the coefficient $d_{j}^{i} \in \mathbb{R}$ have to be determined. In general, three main categories of algorithms can be distinguished: degenerate kernel methods, Nystrom methods, and projection methods. Projection methods can be further subdivided into collocation methods and Galerkin methods. In this paper, for the sake of generality, we apply Galerkin methods.

Due to the approximation of the eigenfunctions stated in Eq. 6, the Fred- 
holm integral equation of Eq. 2 can be solved only approximately. Inserting Eq. 6 into Eq. 2 gives the residual $r_{I E V P}(x)$ :

$$
r_{I E V P}(r)=\sum_{j=1}^{N} d_{j}^{i}\left(\int_{\Omega} \operatorname{Cov}\left(r, r^{\prime}\right) h_{j}\left(r^{\prime}\right) d r^{\prime}-\hat{\lambda}_{i} h_{j}(r)\right)
$$

In Galerkin methods, the coefficients $d_{j}^{i} \in \mathbb{R}$ are chosen such that the residual $r_{I E V P}(r)$ becomes orthogonal to the subspace of $L^{2}(\Omega)$ spanned by the basis functions $\left\{h_{j}\right\}_{j=1}^{N}$. Hence, the following problem is solved:

$$
\int_{\Omega} r_{I E V P}(r) h_{j}(r) d r=0, \forall \mathrm{j}=1, \ldots \mathrm{N}
$$

which can be expressed in matrix notation as the generalized matrix eigenvalue problem:

$$
B d_{i}=\hat{\lambda}_{i} M d_{i}
$$

where B is a symmetric positive semi-definite $N \times N$ matrix whose elements are defined as:

$$
b_{\mathrm{ln}}=\int_{\Omega} h_{l}(r) \int_{\Omega} \operatorname{Cov}\left(r, r^{\prime}\right) h_{n}\left(r^{\prime}\right) d r^{\prime} d r
$$

and $\mathrm{M}$ is a symmetric positive definite $N \times N$ matrix with elements:

$$
m_{\ln }=\int_{\Omega} h_{l}(r) h_{n}(r) d r
$$

As such, the truncated KL expansion can be given by:

$$
\hat{H}(r, \theta)=\mu(r)+\sum_{i=1}^{M} \sqrt{\hat{\lambda}_{i}} \hat{\varphi}_{i}(r) \hat{\xi}_{i}(\theta)
$$


where $\hat{\lambda}_{i}$ and $\hat{\varphi}_{i}$ are approximations to the true eigenvalues $\lambda_{i}$ and eigenfunctions $\varphi_{i}$, and $\hat{\xi}_{i}(\theta)$ are standard uncorrelated random variables, i.e., $E\left[\hat{\xi}_{i}(\theta), \hat{\xi}_{j}(\theta)\right]=\delta_{i j}, \forall i, j \leq M$.

\subsection{Random field propagation}

Because of its matured theoretical background as well as the unique associated computational advantages, the random field has been extensively combined with FEM resulting in an uncertainty analysis framework known as the stochastic FEM (SFEM). The remaining challenge now is to propagate the random field to quantities of interest at the output side of a numerical model $\mathcal{M}$. The most straightforward propagation of a random field is obtained via the Monte Carlo (MC) simulation method. Given a certain quantity of interest $y$ (e.g., the acceleration response of a structure), obtained by propagating $\hat{H}(r, \theta)$ through a deterministic numerical model $\mathcal{M}$, the Monte Carlo estimator is given as:

$$
\begin{aligned}
& \hat{\mu}_{y}=\frac{1}{N} \sum_{i=1}^{N} \mathcal{M}\left(\hat{H}\left(r, \theta_{i}\right)\right) \\
& \hat{\sigma}_{y}^{2}=\frac{1}{N-1}\left(\sum_{i=1}^{N}\left(\mathcal{M}\left(\hat{H}\left(r, \theta_{i}\right)\right)-\hat{\mu}_{y}\right)^{2}\right)
\end{aligned}
$$

with $N$ the number of samples and where the samples of $\hat{H}\left(r, \theta_{i}\right)$ are obtained by sampling from the i.i.d. standard normal variables $\hat{\xi}_{i}$. In this case, it can be shown that the coefficient of variance $(\mathrm{CoV})$ of this estimator

equals $\sqrt{\frac{1-\hat{y}}{\hat{y} \cdot N}}$. As such, in order to obtain a suitable coefficient of variance, MC sampling often comes at a high computational cost. Some extensions and refinements to the conventional MC technique have been proposed and 
they are aimed at directing the sampling to regions of the PDF of highest importance. These techniques include Latin hypercube sampling, adaptive sampling [32], importance sampling [33] or other advanced techniques such as subset simulation [34]. Another common technique of surpassing the high computational cost of MC is obtained by using surrogate models for the representation of the numerical model [35]. To conclude, Fig 1 summarizes the workflow followed in the context of random field analysis.

\section{Interval Field finite element method}

\subsection{General framework}

When the random field is utilized in general engineering practice, there are two essential properties that need to be selected. The first property that should be defined is the distribution of the random field (e.g., Gaussian or non-Gaussian) and the second one is the correlation structure, which is commonly determined by means of a predefined covariance function and its parameters. To make valid assumptions on both properties, the availability of a large amount of samples is usually required. However, such requirement could be very difficult, or even impossible in some cases, to attain.

In an attempt to provide an alternative approach for a representation of the spatial non-determinism under scarce data, Moens et al. [14] introduced the explicit interval field formulation as an interval counterpart to the probabilistic random field framework. The distinctive advantage of the interval field is that it is capable of performing efficient uncertainty analysis with the consideration of spatial dependency of uncertain parameters based on limited information. 


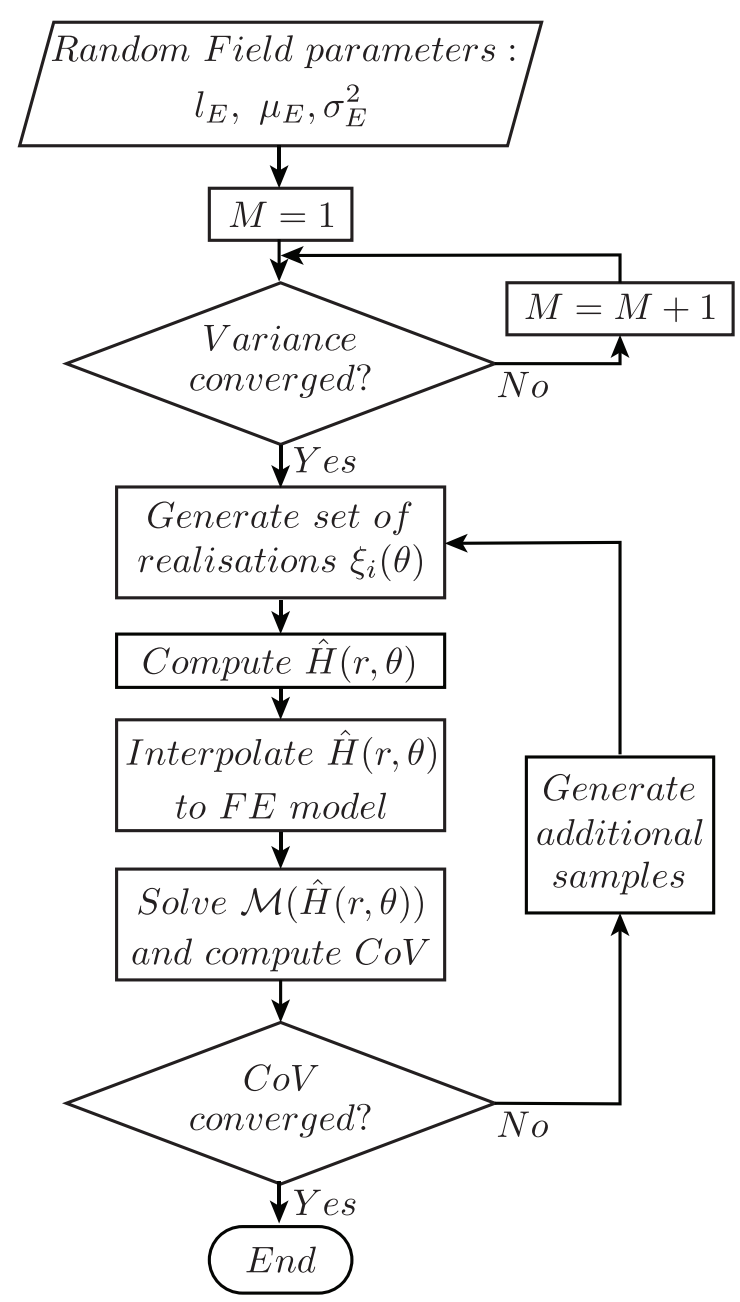

Figure 1: Flowchart of Random Field method

\subsection{Explicit interval field}

The description of an explicit interval field is based on the superposition of $n_{b} \in \mathbb{N}$ base functions $\psi_{i}(r): \Omega \mapsto \mathbb{R}$, scaled by independent interval scalars $\alpha_{i}^{I} \in \mathbb{I} \mathbb{R}$, with $\mathbb{I} \mathbb{R}$ the set of interval scalars. The base functions $\psi_{i}(r)$ describe the spatial nature of the non-deterministic value that is modeled by the interval field over the model domain, and are unit-less. The interval 
scalars $\alpha_{i}^{I}$ on the other hand quantify the bounded non-determinism of the model parameters under consideration. An interval field $x^{I}(r)$ is then formally expressed as:

$$
x^{I}(r)=\mu_{x^{I}}(r)+\sum_{i=1}^{n_{b}} \alpha_{i}^{I} \psi_{i}(r)
$$

with $\mu_{x^{I}}(r)$ the mean trend of the field.

When $\Omega$ is discretized into $\mathrm{k}$ finite elements $\Omega_{e} \subset \Omega$, the discretized base functions $\psi_{i}(r) \in \mathbb{R}^{k}$ interpolate the interval scalars $\alpha_{i}^{I}$ to dependent intervals $x^{I}\left(\Omega_{e}\right)$ for each $\Omega_{e} \subset \Omega$. As such, it is also clear that, when $n_{b}<k$, a reduction of the input space dimension is obtained. Furthermore, since all $\alpha_{i}^{I}$ remain independent, common techniques for the propagation of interval uncertainty can be applied directly.

\subsection{Local interval field decomposition}

For practical applications, the base functions $\psi_{i}(r)$ in Eq. 14 should translate expert knowledge of the analyst on the spatial nature of the uncertainty to a mathematical formulation in an intuitive way, while delivering a realistic representation of this uncertainty. The definition as such can be based either on engineering judgment, or on direct [36] or indirect $[37,38,39]$ measurement data. In the context of the explicit interval field formulation, two techniques exist for the construction of $\psi_{i}(r)$ : Inverse Distance Weighting interpolation [39] and Local Interval Field Decomposition (LIFD) $[40,41]$. In this paper LIFD is applied for the comparison to random field approaches.

Local interval field decomposition (LIFD) starts from the explicit for- 
mulation of the interval field, as introduced in section 3.2. In this approach, the core idea is that the spatial complexity of the interval field realizations is limited by imposing an upper bound on the spatial gradients, as well as a global lower and upper bound on the uncertain properties at each location. For example, in a 1D case, the parameter that represents the spatial dependency in the realizations is the maximum absolute value of the first derivative of the field parameter $\overline{\left|\frac{\partial x}{\partial r}\right|}$. Also, the field parameter itself is bounded by a globally defined minimum and maximum value, $[\underline{x}, \bar{x}]$. This leads to the following restrictions on the realizations $x_{j}^{I}(r)$ :

$$
\begin{aligned}
& x_{j}^{I}(r) \in[\underline{x}, \bar{x}], \forall r \in \Omega \\
& \frac{\partial x_{j}^{I}(r)}{\partial r} \in\left[-\overline{\left|\frac{\partial x}{\partial r}\right|}, \overline{\left|\frac{\partial x}{\partial r}\right|}\right], \forall r \in \Omega
\end{aligned}
$$

To implement this definition of the interval field, LIFD adopts an approach closely related to FEM. An equidistant mesh of points $r_{i}$ is defined, and in each point a piecewise continuous second order polynomial function $\psi_{i}(x)$ is defined as base function, representing a radial basis function with radius $\mathrm{R}$, beyond which the value reduces to zero:

$$
\psi_{i}(r)=\left\{\begin{array}{cc}
0 & r \leq r_{i}-R \\
\frac{2\left(r-r_{i .}+R\right)^{2}}{R^{2}} & r_{i}-R \leq r \leq r_{i}-\frac{R}{2} \\
1-\frac{2\left(r-r_{i .}\right)^{2}}{R^{2}} & r_{i}-\frac{R}{2} \leq r \leq r_{i}+\frac{R}{2} \\
\frac{2\left(r-r_{i .}+R\right)^{2}}{R^{2}} & r_{i}+\frac{R}{2} \leq r \leq r_{i}+R \\
0 & r \geq r_{i}+R
\end{array}\right.
$$

Fig. 2 and Fig. 3 illustrate this concept. 

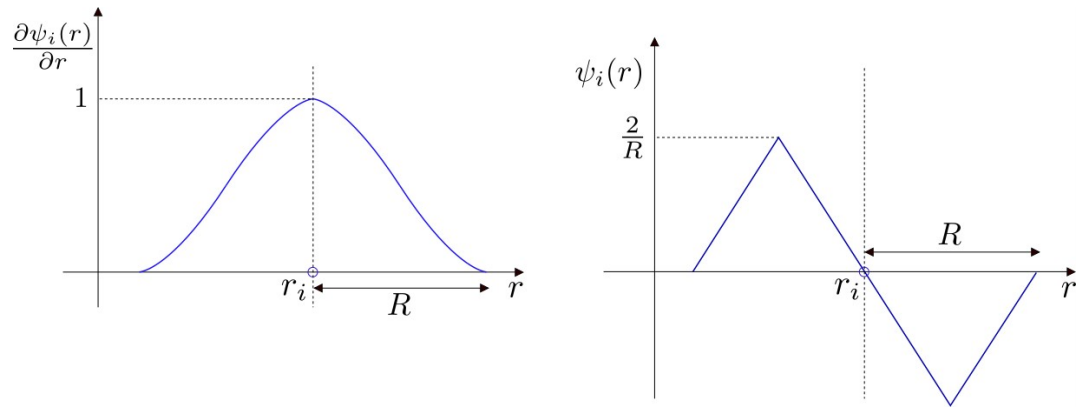

Figure 2: The basis function used in LIFD and its first derivative

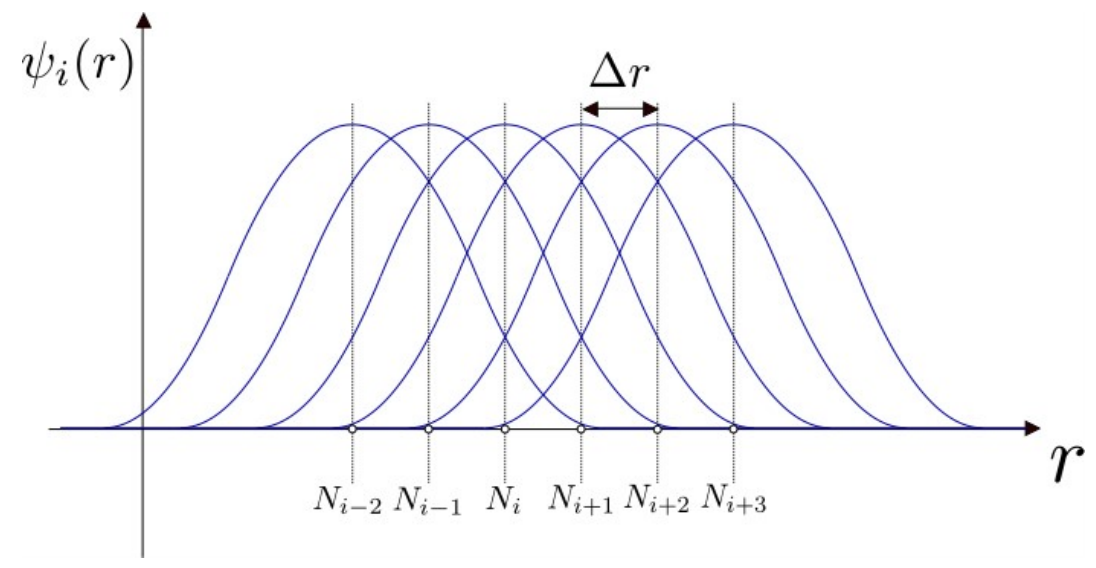

Figure 3: Illustration of the placement of basis functions in an uncertainty mesh

Finally, Eq. 14 transforms into the LIFD interval field as:

$$
x^{I}(r)=C+a \sum_{i=1}^{n_{b}} \alpha_{i}^{I} \psi_{i}(r)
$$

In this equation, all interval scalars $\alpha_{i}^{I}$ reduce to independent unitary intervals $[-1,1]$. The governing parameters of the LIFD are the scaling factor $a$ (which is constant for a homogeneous field), offset $C$, basis function radius $R$ and mesh distance $\Delta x=r_{i+1}-r_{i}$. The relations to the global uncertainty 
parameters are as follows:

$$
\begin{aligned}
& \overline{\left|\frac{\partial x}{\partial r}\right|}=\frac{2 a}{\Delta x} \\
& \bar{x}=C+\frac{a R}{\Delta x} \\
& \underline{x}=C-\frac{a R}{\Delta x}
\end{aligned}
$$

Using these relations, the realizations obey the maximum gradient as well as global interval constraints.

The mesh size can be chosen to be small or large depending on the local variations that are represented by the field. Smaller mesh sizes lead to higher dimensions as more basis functions and allocated intervals are needed, but it allows for the representation of smaller local variations. This is comparable to including more or less basis functions in a $\mathrm{KL}$ decomposition, as the higher order basis functions exhibit more local differences. In theory, the mesh size can be chosen equal to the FE mesh, putting a basis function in every element of the FE mesh. This includes every possible realization that obeys the maximum gradient constraint into the uncertain set, at the cost of a potentially very high dimensionality. Since the dimensionality greatly affects the computational effort of the analysis, a tradeoff has to be made between resolution of local variability and computational resources.

If a set of measurement data is available, the LIFD can be applied to infer the global uncertainty parameters from the data while keeping the intervals independent. Recently, an application to construct such base functions from a limited set of measurement data was introduced [36]. 


\subsection{Interval field propagation}

The solution of the numerical model $\mathcal{M}$ containing interval field uncertain parameters $x^{I}(r)$ is aimed at finding those realizations of the interval field that yield extrema in the set of model responses. In the case there are $d$ quantities of interest $y_{i}$, this set is expressed for each individual element as:

$$
\tilde{y}_{k}=\left\{y_{k} \mid y_{k}=\mathcal{M}_{k}\left(x_{j}^{I}(r)\right), \forall x_{j}^{I}(r) \in x^{I}(r)\right\}, k=1 \ldots d
$$

It is clear that through the definition of the interval field $x^{I}(r)$ as specified in the previous section, this comes down to finding the output set for a hypercubic input domain spanned by the independent interval scalars $\alpha_{i}^{I}$. This output set can therefore be obtained using one of the classical multivariate interval finite element analysis methods such as interval arithmetic, a global optimization approach or perturbation methods [1].

In this paper, the global optimization is used for propagation. Global optimization techniques aim at finding the smallest conservative hypercube approximation $y^{I}$ of $\tilde{y}[5]$. This optimization algorithm is explicitly given as:

$$
\begin{aligned}
& y_{\underline{k}}=\min _{x_{j}^{I}(r) \in x^{I}(r)} \mathcal{M}_{k}\left(x_{j}^{I}(r)\right), k=1, \ldots d \\
& \bar{y}_{k}=\max _{x_{j}^{I}(r) \in x^{I}(r)} \mathcal{M}_{k}\left(x_{j}^{I}(r)\right), k=1, \ldots d
\end{aligned}
$$

Generally, the optimization method has a high computational cost. Therefore, meta-models are commonly used for the representation of the determinis1tic numerical model. In this context, response surface models [42], radial basis functions [43], Artificial Neural Networks [44], Support Vector Machines [45] as well as Kriging [46, 47] has been applied. A recent comparison of several machine learning methods in an engineering context is 
given by [48]. Based on the findings in this paper, Kriging is used in this work The latter is used in this work. To conclude, the workflow to define and propagate interval fields is illustrated in Fig 4.

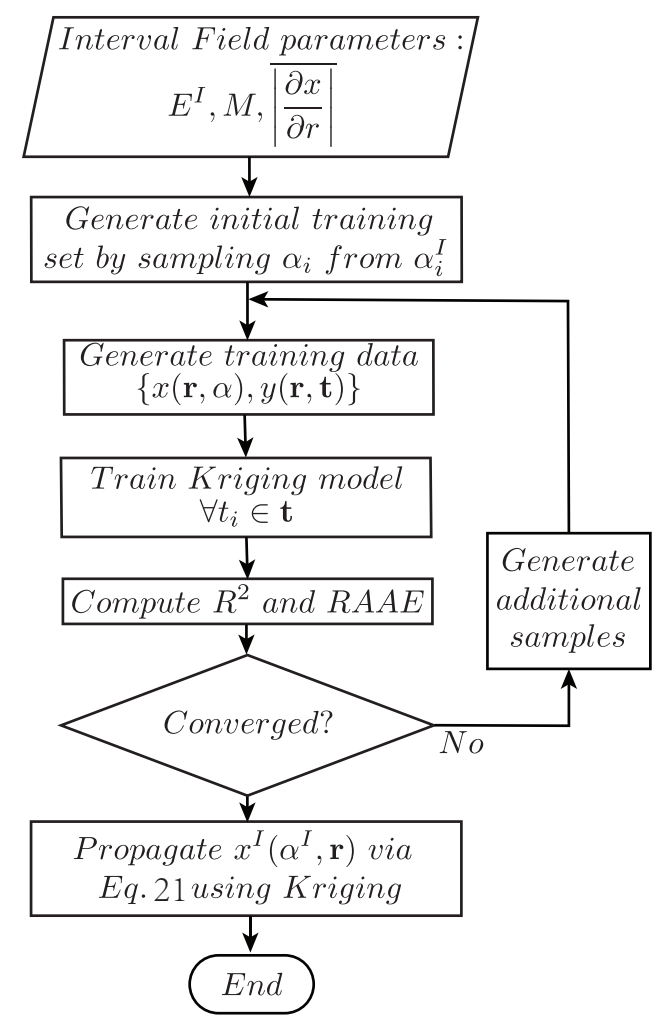

Figure 4: Flowchart of Interval Field method

\section{Deterministic landing dynamic analysis for a lunar lander}

\subsection{Finite element model of a lunar lander}

In this section, a finite element model of a typical legged lunar lander is built, which is composed of three basic components: (1) the center body, (2) solar wings, and (3) the landing gears. For the sake of simplification, 
the connection between center body and landing gear is replaced by twelve equivalent spherical joints, through which the buffer loads generated from compression and stretching of the landing gears are transferred to the center body during the soft landing of the lunar lander. The center body and two solar wings are connected by spring-hinges. In the simplified model, the main structures of the center body and the solar wings are retained. The payloads and auxiliary equipment are simplified as the distributed inertia adhered to the original position.

The solar wing is an important part of the equipment on the lunar lander. It is made of laminated composite plates and its ply orientation angle, ply thickness and ply stacking sequence have been determined through an optimal design process. In this example, the solar wing is modeled as a composite shell with 16 layers and the orientation angle of each layer is $[0 / 45 / 45 / 90]_{4 s}$. Each layer has the same material (carbon fiber M60JB) and same thickness $(0.05 \mathrm{~mm})$.

The final finite element model for analysis is shown in Fig. 5. The effectiveness of the proposed simplified model has been validated by comparison with test data, the error between the result of simulation and test data is less than $5 \%$, see [24]. The weight of the total structure is $1050 \mathrm{~kg}$ and the FE model is meshed by 15900 quadrilateral elements. The mass of the solar wing is only $4.4 \mathrm{~kg}$, so a small uncertainty in the wing's properties has almost no impact on the center body response. Nonetheless, it will affect the response dynamics of the solar wing.

To analyze the dynamic response of the lunar lander during a landing procedure, we simulate the lunar lander falling vertically from a height of $0.2 \mathrm{~m}$ at a speed of $4 \mathrm{~m} / \mathrm{s}$. The response point is selected on the edge of 


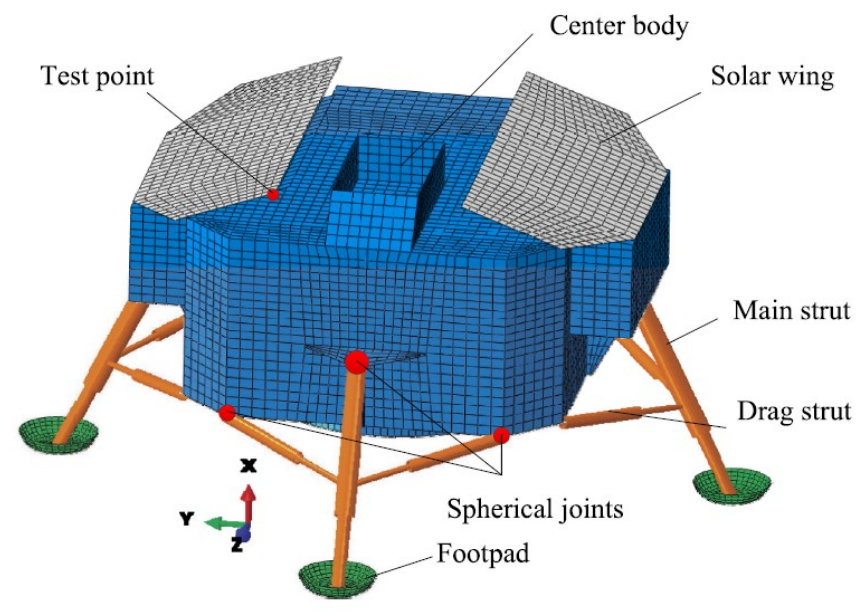

Figure 5: Finite element model of the lunar lander[24]

the left solar wing, as shown in Fig. 5. The analysis takes 200 steps and increment time is $0.001 \mathrm{~s}$, so the total analysis time is $0.2 \mathrm{~s}$.

\subsection{Impulse based substructuring method}

Since the lunar lander is a large-scale model, substructuring technology is used to improve the efficiency. In this example, we only consider the uncertainty of solar wing material properties. So we divide the whole model into two substructures: the center body and the solar wing and we use substructure technology to solve the response. The center body is translated to an Impulse Based Substructure (IBS) while the solar wing remains a finite element substructure. By using IBS, the dynamic response of the center body is described by the convolution of the Impulse Response Function (IRFs) matrix $H(t)$ and the applied force vector $f(t)$ (i.e. the Duhamel's integral). The displacement vector $u$ of the center body substructure $\mathbf{s}$ at 
time $\mathbf{t}$ can be written as:

$$
u^{(\mathbf{s})}(\mathbf{t})=\int_{0}^{\mathbf{t}} \mathrm{H}^{(\mathbf{s})}(\mathbf{t}-\tau) f^{(\mathbf{s})}(\tau) d \tau
$$

where superscript $\mathbf{s}$ denotes the serial number of the substructures. Applying the trapezoidal rule to the Duhamel's integral, the finite difference form of the displacement can be described approximately as:

$$
u_{\mathbf{n}}^{(\mathbf{s})}=\sum_{\mathbf{i}=0}^{\mathbf{n}-1} \mathrm{H}_{\mathbf{n}-\mathbf{i}}^{(\mathbf{s})}\left(f_{\mathbf{i}}^{(\mathbf{s})}+f_{\mathbf{i}+1}^{(\mathbf{s})}\right) \frac{d \mathbf{t}}{2}
$$

The equations of motions of the solar wing finite element substructure $r$ can be described as:

$$
M^{(r)} \ddot{u}^{(r)}(t)+C^{(r)} \dot{u}^{(r)}(t)+K^{(r)} u^{(r)}(t)=f^{(r)}(t)+g^{(r)}(t)
$$

where $f^{(r)}(t), g^{(r)}(t)$ are the external forces and interface forces acting on FE substructure $r$.

To assemble the substructures, the interface displacement compatibility and interface force equilibrium are introduced. The displacement compatibility is described as:

$$
B^{(r)} u^{(r)}+B^{(s)} u^{(s)}=0
$$

where $B$ is a signed Boolean matrix identifying the matching degrees of freedoms (DOFs) on the interfaces.

The force equilibrium requires that interface forces on each side of the interface between any two connected substructures are an equilibrating force system. For matching interface assembly, the interface forces should comply 
the Newton's third law. This can be described as:

$$
\begin{aligned}
& g^{(\mathbf{s})}=B^{(\mathbf{s})^{\mathrm{T}} \lambda} \\
& g^{(\mathbf{r})}=B^{(\mathbf{r})^{\mathrm{T}} \lambda}
\end{aligned}
$$

where $g^{(\mathbf{s})}$ denotes the full set of interface forces of center body substructure $\mathbf{s}, g^{(\mathbf{r})}$ denotes the full set of interface forces of solar wing substructure $\mathbf{r}, \lambda$ is the Lagrange multipliers vector.

Through the Newmark- $\beta$ scheme, the finite difference form of Eq. 24 can be described as:

$$
u_{n}^{(r)}=R_{n}^{(r)}+\Delta t^{2} \beta^{(r)^{-1}} B^{(r) \mathrm{T}} \lambda_{n}
$$

where

$$
\left\{\begin{array}{l}
\underline{M}^{(r)}=M^{(r)}+C^{(r)} \gamma \Delta t+K^{(r)} \Delta t^{2} \beta \\
R_{n}^{(r)}=\underline{u}_{n}^{(r)}+\Delta t^{2} \beta \underline{M}^{(r)^{-1}}\left(f_{n}^{(r)}-K^{(r)} u_{n}^{(r)}-C^{(r)} \dot{u}_{n}^{(r)}\right)
\end{array}\right.
$$

and

$$
\left\{\begin{array}{l}
\underline{\dot{u}}_{n}^{(r)}=\dot{u}_{n-1}^{(r)}+\Delta t(1-\gamma) \ddot{u}_{n-1}^{(r)} \\
\underline{u}_{n}^{(r)}=u_{n-1}^{(r)}+\Delta t \cdot \dot{u}_{n-1}^{(r)}+\Delta t^{2}(1 / 2-\beta) \ddot{u}_{n-1}^{(r)}
\end{array}\right.
$$

Combining displacement compatibility and substituting Eq. 26 into Eq. 23 and Eq. 27 yields the system governing equation:

$$
\left\{\begin{array}{l}
u_{n}^{(r)}=R_{n}^{(r)}+\Delta t^{2} \beta \underline{M}^{(r)^{-1}} B^{(r) \mathrm{T}} \lambda_{n} \\
u_{\mathbf{n}}^{(\mathbf{s})}=\sum_{\mathbf{i}=0}^{\mathbf{n}-1} H_{\mathbf{n}-\mathbf{i}}^{(\mathbf{s})}\left[f_{\mathbf{i}}^{(\mathbf{s})}+f_{\mathbf{i}+1}^{(\mathbf{s})}+B^{(\mathbf{s}) \mathrm{T}} \lambda_{\mathbf{i}}+B^{(\mathbf{s}) \mathrm{T}} \lambda_{\mathbf{i}+1}\right] \frac{d \mathbf{t}}{2} \\
B^{(r)} u_{\mathbf{n}}^{(r)}+B^{(s)} u_{\mathbf{n}}^{(\mathbf{s})}=0
\end{array}\right.
$$


By solving Eq. 30, the Lagrange multipliers at time $t$ can be found as:

$$
\lambda_{\mathbf{n}}=-\left(B^{(\mathbf{s})} H_{1}^{(\mathbf{s})} B^{(\mathbf{s})^{T}}+B^{(r)} \beta \Delta t^{2}\left(\underline{M}^{(R)}\right)^{-1} B^{(r) \mathrm{T}}\right)^{-1}\left(B^{(\mathbf{s})} \tilde{u}_{\mathbf{n}-1}^{(\mathbf{s})}+B^{(r)} R_{n-1}^{(R)}\right)
$$

where

$$
\begin{aligned}
\tilde{u}_{\mathbf{n}-1}^{(\mathbf{s})}= & \sum_{\mathbf{i}=0}^{\mathbf{n}-2} \mathrm{H}_{\mathbf{n}-1}^{(\mathbf{s})}\left[f_{\mathbf{i}}^{(\mathbf{s})}+f_{\mathbf{i}+1}^{(\mathbf{s})}+G_{\mathbf{f}}^{(\mathbf{s})} B^{(\mathbf{s})^{T}}\left(\lambda_{\mathbf{i}}+\lambda_{\mathbf{i}+1}\right)\right] \\
& +H_{1}^{(\mathbf{s})}\left(f_{\mathbf{n}-1}^{(\mathbf{s})}+f_{\mathbf{n}}^{(\mathbf{s})}+G_{\mathbf{f}}^{(\mathbf{s})} B^{(\mathbf{s})^{T}} \lambda_{\mathbf{n}-1}\right)
\end{aligned}
$$

Substituting $\lambda_{\mathbf{n}}$ into Eq. 30 results in $u_{\mathbf{n}}^{(\mathbf{s})}$. Then, $\dot{u}_{\mathbf{n}}^{(\mathbf{s})}$ and $\ddot{u}_{\mathbf{n}}^{(\mathbf{s})}$ can be obtained through the Newmark- $\beta$ scheme. In this work, we use $\gamma=0.5$ and $\beta=0.25$ for default.

In Eq. $31, B^{(s)}, B^{(r)}$ and $H^{(s)}$ are constant and only need to be calculated one time. During repeated analysis, each time we only need to calculate the M, C, and K matrix of the solar wing and substitute them into Eq. 28 in order to obtain $\lambda_{\mathbf{n}}$ and $u_{\mathbf{n}}^{(\mathbf{s})}$. Overall, this IBS approach reduces the simulation time from 1 hour for a full FEM analysis to only 1 minute.

The comparsion between the IBS method and the finite element model reference result is shown in Fig. 6.the resulting deterministic acceleration and velocity response of the test point of Fig. 5 are shown in Fig. 6. The results show that the IBS method has high accuracy. Furthermore, it should be noted that during the landing procedure, the maximum acceleration of the response point is about $80 \mathrm{~g}$. 


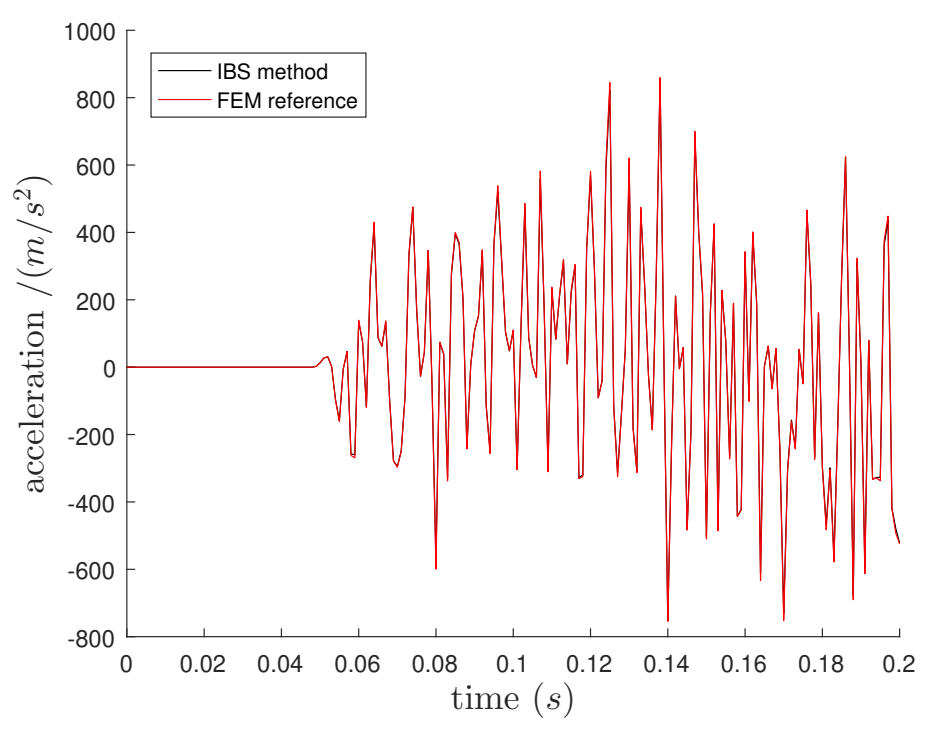

(a) Acceleration in the test point

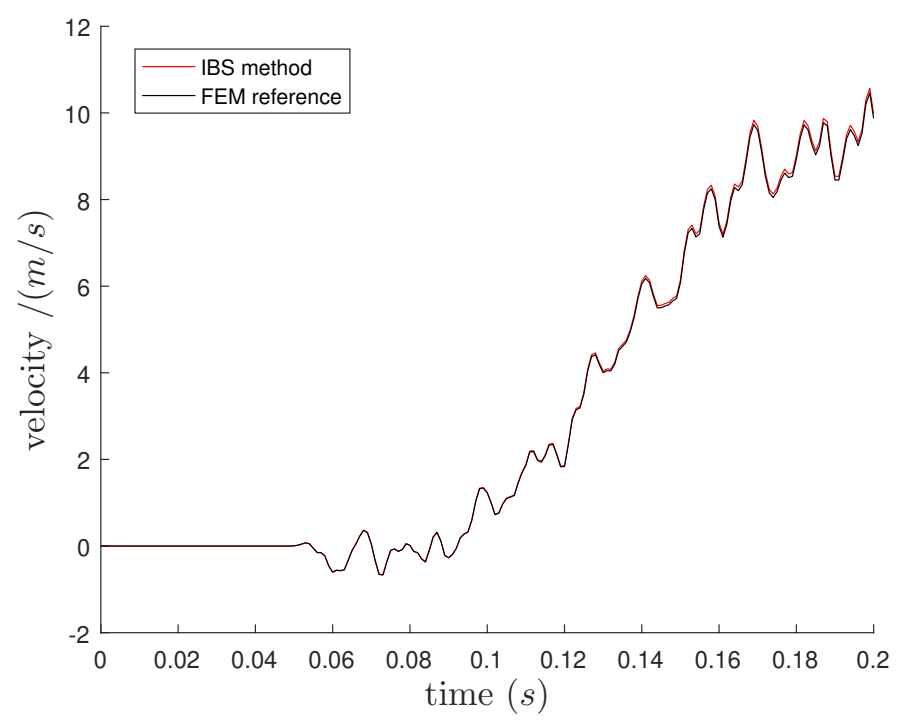

(b) Velocity in the test point

Figure 6: Dynamic results of the lunar lander using Impulse Based Substructuring

\section{Uncertain fields at the input}

In this section, the lunar lander dynamic FE model is used as a case study to compare interval fields, gę6erated based on the LIFD method, 
with Gaussian random fields in terms of the realizations that are generated at the input side of the model. Specifically, Young's modulus of the composite panels of the solar wings are considered to be subjected to spatial uncertainty. For illustrational reasons, and without loss of generality, we consider that this uncertainty is dominant in one specific direction, indicated by $r$ as illustrated in Fig. 7 .

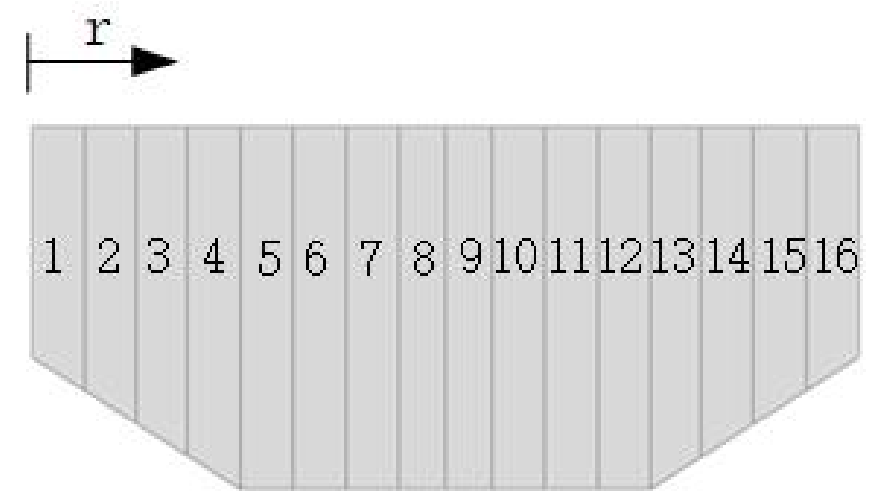

Figure 7: 1-D spatial field orientation and discretization of the solar wing

In the $r$ direction, the total length of solar wing is $2.5 \mathrm{~m}$, spanning 32 elements in the model. To generate the discretized random and interval fields, the solar wing is subdivided into 16 zones along $r$. The material properties within such a zone are used for all elements within that zone. The numbers corresponding to these zones are also illustrated in Fig. 7.

We now first define the random fields according to the state-of-the-art approach in literature, based on an assumed correlation kernel. In a second step, the interval field representation is generated using the LIFD approach based on global field and gradient bounds obtained from a large sample set of the random field. This should allow for an objective comparison between 27 
the two modeling strategies, as they both refer to the same underlying realization set.

\subsection{Random field and interval field modeling}

Random field modeling: An isotropic Gaussian random field with squared exponential covariance kernel with a correlation length spanning $l_{E}=8$ elements (which corresponds to $0.6 \mathrm{~m}$ ) is considered in this example. The mean of the random field is taken to be $146 \mathrm{GPa}$ and the variance 7.3 GPa, which corresponds to a coefficient of variance of $5 \%$. Based on the convergence of the variance error, the KL series expansion is truncated after $M=6$ terms.

Since Young's modulus is considered to be constant over two neighboring elements, this random field can be interpreted as a 16-dimensional multivariate normal distribution, where each random variable corresponds to a zone of constant Young's modulus in the FE model. One random field realization of Young's modulus of the solar wing is shown in Fig. 8.

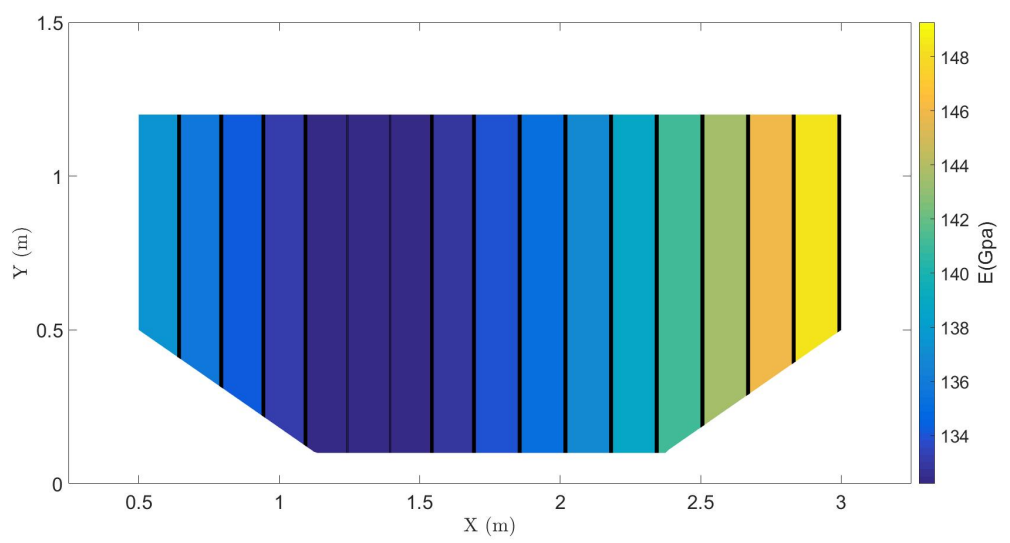

Figure 8: Random field realization of the E-modulus in the solar wing 
Interval field modeling: The interval field on the other hand is constructed using the local interval field decomposition, as explained in section 3.3. Special care is taken in the definition of the interval field to allow a direct comparison to the random field:

- Global interval bounds: the global upper and lower bound of the interval field are chosen as $E^{I}=[124,168] \mathrm{GPa}$, which corresponds to the $\left[\mu_{E}-3 \sigma, \mu_{E}+3 \sigma\right]$ bounds of the random field. These bounds are taken to be homogeneous over $\Omega$, although this is not a necessary condition for the definition of an interval field.

- Series expansion truncation: since the KL expansion of the random field is truncated after 6 terms, also the interval field series expansion is truncated after 6 terms. In practice, this means that the amount of independent interval variables in the interval field series expansion is equal to the amount of standard normal random variables in the random field series expansion. Therefore, the uncertain dimension of both uncertain field models is considered to be equal. Note that obviously the information contained in both uncertain spaces is fundamentally different.

- Basis functions: the core of the idea on how to compare interval fields with random fields lies in the definion of the LIFD basis functions. As is clear from Eq. 17 to Eq. 23, an important property of the LIFD basis is the maximum absolute value of the first derivative of the field parameter $\overline{\left|\frac{\partial x}{\partial r}\right|}$. Therefore, as a first step in the LIFD basis, the maximum absolute value of the first derivative of the field parameter 
$\overline{\left|\frac{\partial x}{\partial r}\right|}$ is computed over a set of realizations of the random field:

$$
\overline{\left|\frac{\partial x}{\partial r}\right|}=\max _{j=1, \cdots, N}\left|\frac{\partial \hat{H}\left(r, \theta_{j}\right)}{\partial r}\right|
$$

with $\theta_{j}$ representing $N$ stochastic samples of the random field such that appropriate convergence in a stochastic sense is obtained. Then, based on this computed quantity, the LIFD basis is constructed using the relations in Eq. 17 to Eq. 23. Specifically for this case, a radial basis radius $R=10$ elements, mesh distance $\Delta x=5$ elements and scaling factor $a=0.075$ are selected. These basis functions provide, as explained in Section 3.3, a functional mapping of a 6-dimensional hypercube to a 16-dimensional polytopic domain where each dimension corresponds to a zone of constant Young's modulus in the solar wing. One such realization of the interval field is illustrated in Fig. 9.

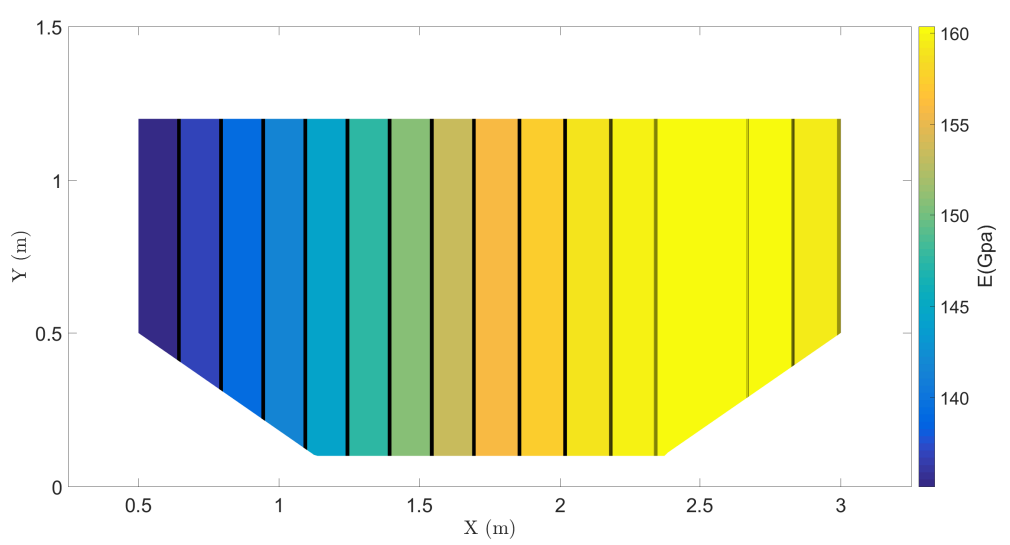

Figure 9: Interval field realization of the E-modulus in the solar wing 


\subsection{Comparison of interval field and random field realizations}

This section aims at a thorough comparison of the realizations that are provided by both the interval and the random field, as described in the previous section. The realizations of these two field concepts are first interpolated to the 16 zones of constant Young's modulus of the model under consideration, based on the respective methods that are described in sections 2 and 3 . Both sets of realizations are conceptually different. The interval field on the one hand can intuitively be represented as a 16dimensional convex hull, since the basis functions $\psi_{i}$ project the $n_{b}$ interval scalars $\alpha_{i}$ towards an interval corresponding to each of the zones. Note that these intervals are not independent from each other, but coupled by means of the aforementioned basis functions. Hence, a convex hull representing a set of jointly-admissible values within the intervals is generated [49].

A random field on the other hand can, after discretization, be represented as a multivariate normal distribution where each random variable corresponds to a zone of constant Young's modulus, and where the correlation between these random variables depends on the auto-correlation function and corresponding correlation length. As such, both field concepts translate a limited set of non-deterministic scalars - uncorrelated random variables / independent intervals - that are globally defined towards nondeterministic yet correlated / dependent local material properties.

The equivalence between both approaches is further illustrated in Fig. 10. This figure shows two-dimensional intersections of the convex hull of the interval field as well as the multivariate normal distribution of the random field for a selection of 10 zones of the model. In this figure, the interval field realizations are illustrated (in red) as the 16-dimensional convex hull 
that corresponds to sampling the bounds of the interval scalars in Eq. 18. First, it can be noted that by tuning the global as well as gradient bounds in the interval field towards the realization set of the random field, a large similarity is obtained between the non-determinism of the local properties in both field models, both in terms of spanned area as well as in mutual interactions. In practice, this means that the auto-dependence in an interval field is mostly dictated by the allowed gradients in the basis functions. In fact, this observation is logical, since by imposing a limit on the absolute value of the maximum gradient, the highest allowed rate of change between two neighboring points is limited. Loosely speaking, a similar situation happens when the correlation length is increased. Indeed, when a high correlation length is imposed on the auto-correlation function of a random field, the gradients of the realizations of the random field decrease since the corresponding random variables have a much higher degree of correlation. Further, by tuning the interval bounds towards the $\pm 3 \sigma$ bounds of the random fields, it is also clear that the magnitude of the uncertainty that is captured by both field representations is comparable. Note that not all realizations of the random field are captured by the interval field. This is a direct result of the infinite support of the Gaussian distribution that underlies the random field, as compared to the crisp boundaries on the possible local values that are imposed by the global bounds of the interval field.

As a means of further comparison, both the convex hull over the interval field realizations and the joint distribution that represents the set of random field realizations are decomposed into their principal components. In case of the random field, this corresponds to the eigenvalues and eigenfunctions of the random field $\hat{H}(r, \theta)$. As concerns the interval field, a singular value 


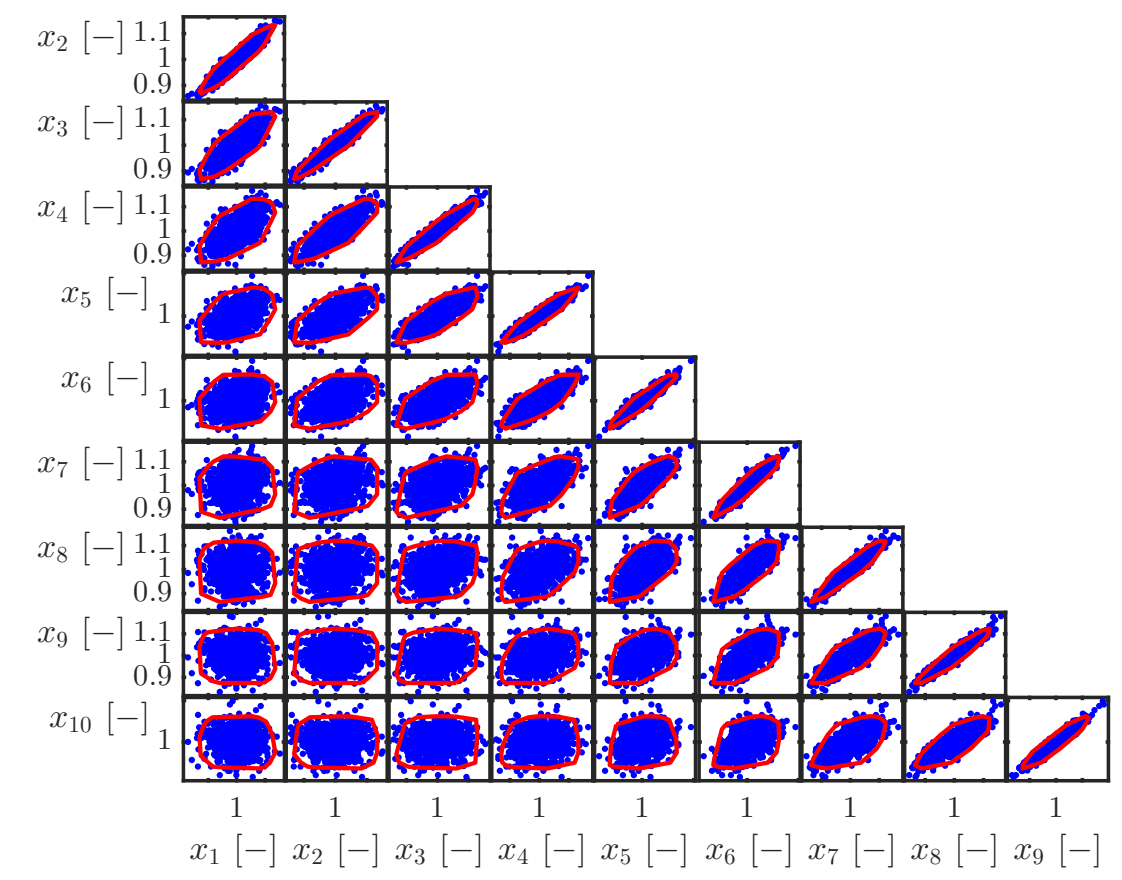

Figure 10: Two-dimensional intersections of a ten dimensional subset of the input space: convex hull representing the interval field (in red), point cloud corresponding to the random field (in blue)

decomposition over the joint covariance matrix is performed as:

$$
\Xi=\Phi \Lambda \Phi^{T}
$$

with $\boldsymbol{\Lambda} \in \mathbb{R}^{16 \times 16}$ the diagonal matrix of the ordered eigenvalues $\lambda_{1} \leq \lambda_{2} \leq$ $\cdots \leq \lambda_{16}$ of $\boldsymbol{\Xi}$, and $\boldsymbol{\Phi} \in \mathbb{R}^{16 \times 16}$ a matrix containing the orthogonal eigenvectors $\phi_{j} \in \mathbb{R}^{16}, j=1, \ldots, 16$. The covariance matrix $\boldsymbol{\Xi}$ is fitted using empirical covariance estimation methods on a set of 264 realizations of the interval field, obtained by means of a uniform sampling between the bounds of the interval scalars $\alpha_{i}^{I}$ shown in Eq. 18. It should be stressed that it is not 
intended to derive a probabilistic description of this non-probabilistic quantity. Rather, this singular value decomposition serves as a linear principal component analysis to compare how the interval field realizations behave with respect to those of the random field as concerns their auto-dependence structure.

Fig. 11 shows the eigenvalues of the random field, compared to the eigenvalues of the covariance matrix fitted to realizations of the interval field, as obtained by solving Eq. 34. As can be noted, they show a similar decay with respect to the eigenvalue number, indicating that both sets of realizations have a similar number of principal directions in their uncertainty space. Further, Fig. 12 shows that also the eigenfunctions of the random field are highly comparable to the eigenvectors that are obtained by performing a singular value decomposition on the auto-covariance matrix that is derived from the interval field realizations. Both observations show that, by tuning the gradient of the interval field basis functions towards the random field realization set, a highly comparable measure for spatial dependence in random and interval fields can be obtained, and that in fact, the vector basis that is used to build both sets of realizations is almost equal. This observation is far from obvious, as the actual basis functions of the interval field, being overlapping radial basis functions, are not orthogonal themselves. Hence, the maximum gradient in the LIFD technique as introduced above is comparable to the concept of a correlation length in random fields.

\section{Dynamic response comparison}

This section discusses the propagation of the previously introduced random and interval fields for the spatial uncertainty in Young's modulus in 


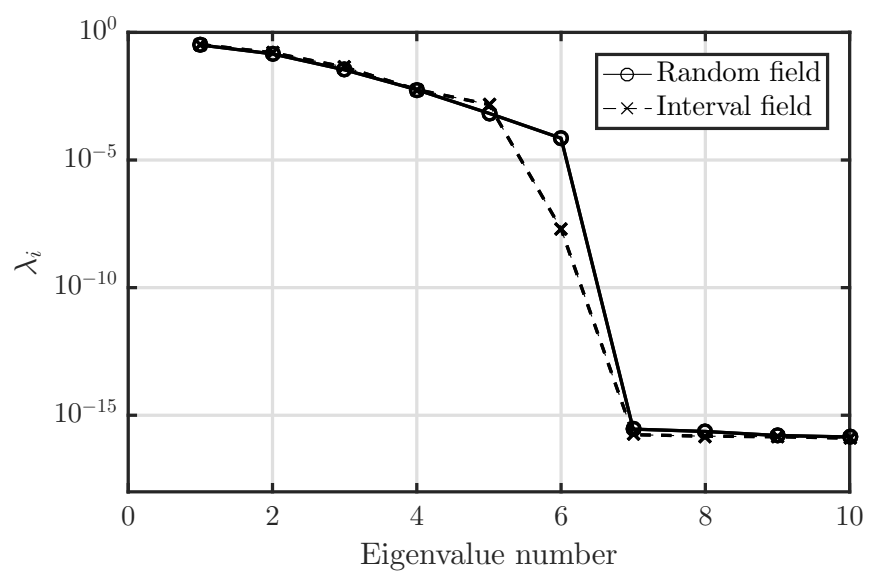

Figure 11: Eigenvalues of random field and interval field covariance kernels

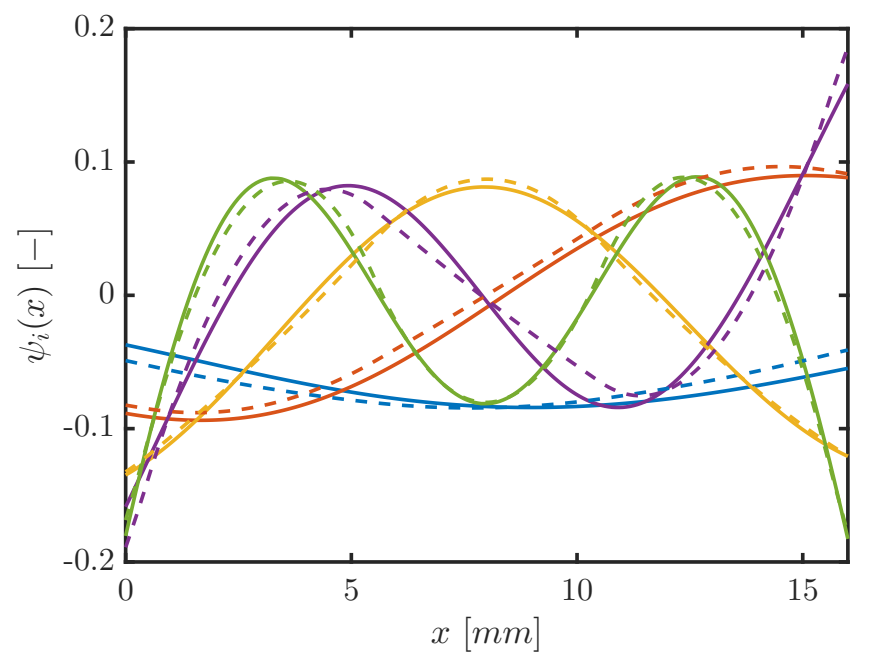

Figure 12: Eigenvector of random field (full) and interval field (dashed)

the solar wing towards the dynamics of the lunar lander during the landing simulation. Appropriate UQ methods are combined with the Impulse Based Substructuring that is presented in Section 4. Then, the response of the solar wing as a result of both modeling strategies is discussed. 


\subsection{Uncertainty analysis based on random field propagation}

The random field model is propagated using Monte Carlo simulation (see Eq. 13). First, we conduct simulations with $N=100,200,300,400,500,1000$ and 1500 samples and the Coefficient of Variation (COV) of the Monte Carlo simulation is used to assess the convergence of the Monte Carlo estimators (see Section 2.3).

Fig. 13 illustrates the convergence of the COV of the acceleration response at several time steps during the time history, as a function of the sample size of the Monte Carlo calculation. Based on this figure, the random field will be propagated in the remainder of the study using 1000 samples. Fig. 14 shows the result of the random field propagation to the acceleration

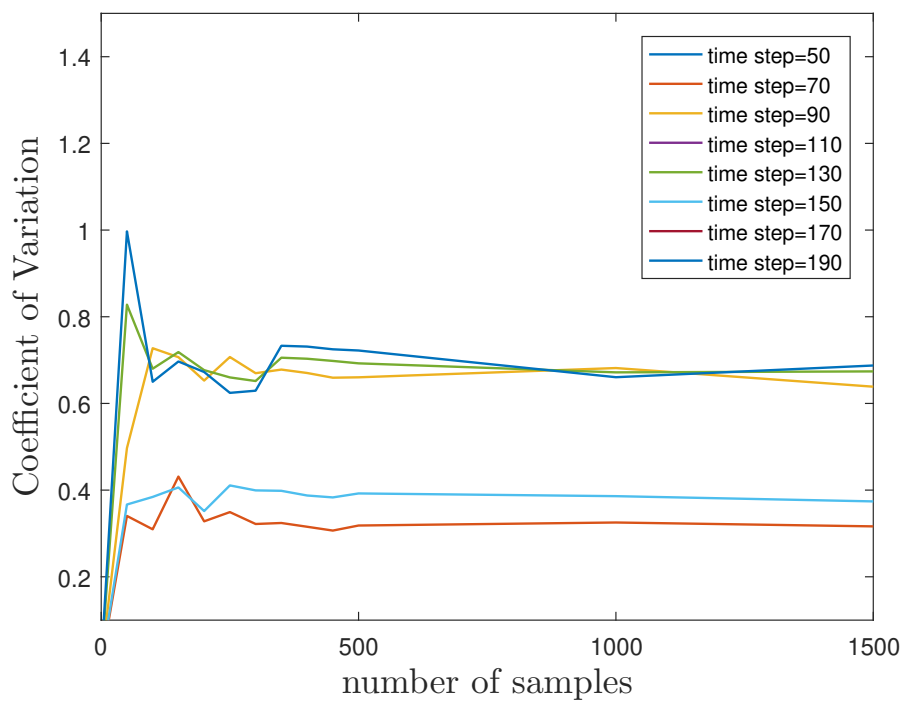

Figure 13: Coefficient of Variation of acceleration response

and velocity of the test point on the solar wing during the landing procedure. The red lines represent the $\left[\mu_{y}-3 \sigma, \mu_{y}+3 \sigma\right]$ interval bounds which 
are compared to the interval field bounds in the next section, and the yellow lines represent 1000 Monte Carlo simulations.

\subsection{Uncertainty analysis based on interval field propagation}

As mentioned in section 5, the interval field $x^{I}(r)$ translates a 6-dimensional hypercube to a 16-dimensional convex hull, where each dimension of the hull corresponds to a location in the FE mesh. This interval field $x^{I}\left(\alpha^{I}, r\right)$ is propagated towards the extremes of the velocity and acceleration of the solar wing at each time instant $t_{i} \in\left[0, t_{\text {end }}\right]$ by means of a global optimization strategy:

$$
\begin{aligned}
& \underline{y}\left(r, t_{i}\right)=\min _{\alpha_{k} \in \alpha^{I}} \mathcal{M}\left(x^{I}\left(\alpha_{k}, r\right), t_{i}\right) \quad i=1, \cdots, n_{t} \\
& \bar{y}\left(r, t_{i}\right)=\max _{\alpha_{k} \in \alpha^{I}} \mathcal{M}\left(x^{I}\left(\alpha_{k}, r\right), t_{i}\right) \quad i=1, \cdots, n_{t}
\end{aligned}
$$

with $\alpha_{k}$ realizations of the interval vector $\alpha^{I} \in \mathbb{I R}^{6}$ and $n_{t}$ the total number of considered time instants $t_{i}$ in the time history of the dynamic simulation. The explicit dependence of the interval field is added to the notation to illustrate that the propagation of the interval field indeed comes down to optimizing the response of the structure with respect to the bounds that are imposed on the 6 global interval scalars.

The model consists of 200 time steps for the velocity and acceleration profiles. For such long time signals, a global optimization might prove to be computationally intractable. Therefore, a Kriging surrogate model is constructed for each time step. To train this Kriging model, a training set of $\left(x^{I}\left(\alpha_{k}, r\right), y_{i}\left(r, t_{i}\right)\right), t_{i} \in\left[0, t_{e n d}\right]$ parameter pairs is generated based on a Sobol set of $n_{\text {Sobol }}$ samples combined with the $q=2^{6}$ vertices of the hyper-cubic input space modeled by the global interval vector $\alpha^{I}$.

In order to validate the Kriging model for different Sobol set sample sizes 


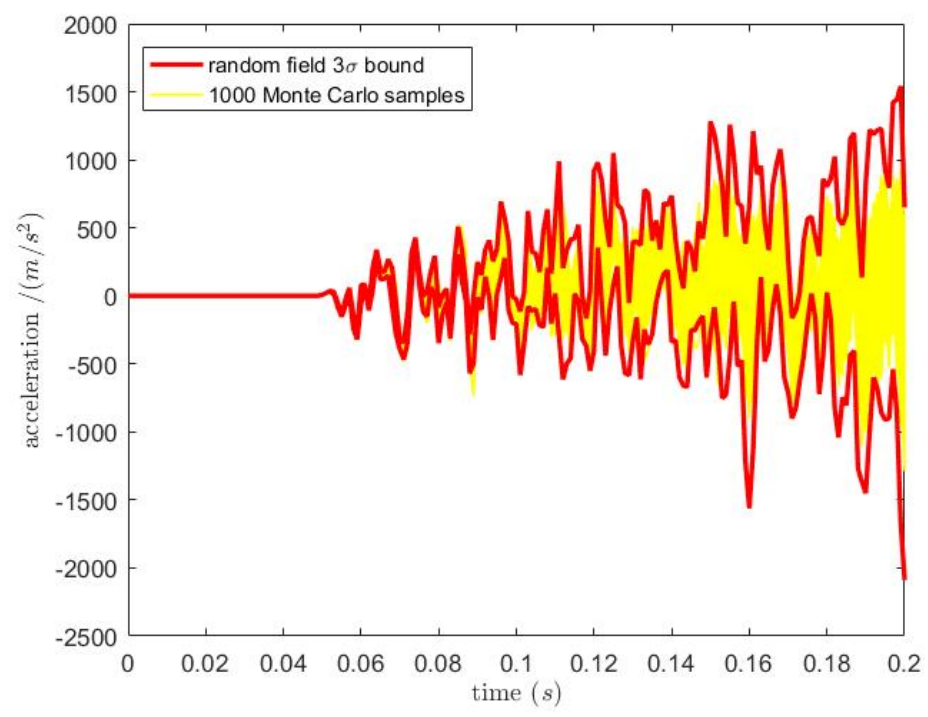

(a) $3 \sigma$ bounds on the acceleration response compared to the Monte Carlo samples

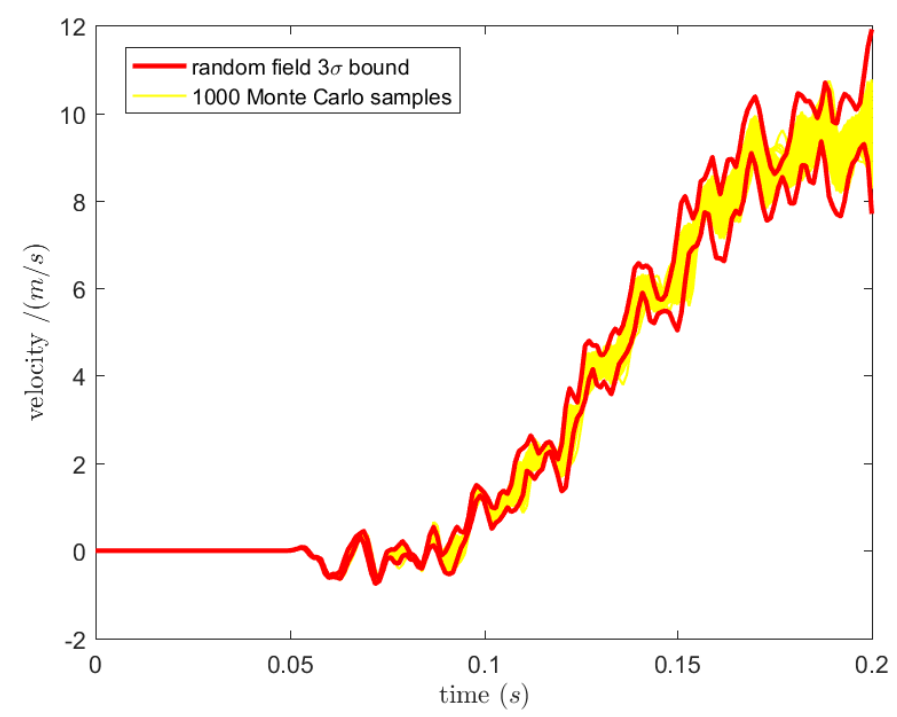

(b) $3 \sigma$ bounds on the velocity response compared to the Monte Carlo samples

Figure 14: Lower and upper $3 \sigma$ bounds on the acceleration and velocity response of the solar wing solved using random fields 
$n_{\text {Sobol }}$, we take $n_{v a l}=20$ samples within the parameter range, and construct realizations of the input parameter field. We calculate the acceleration for these fields both by FEM simulation and the Kriging model, after which the $R^{2}$ and the Relative Average Absolute Error (RAAE) [50] values are computed at each time instant using:

$$
\begin{aligned}
& R^{2}\left(t_{i}\right)=1-\frac{\sum_{j=1}^{n_{\text {val }}}\left(y_{j}\left(t_{i}\right)-\hat{y}_{j}\left(t_{i}\right)\right)^{2}}{\sum_{j=1}^{n_{\text {val }}}\left(y_{j}\left(t_{i}\right)-\mu_{y_{j}}\left(t_{i}\right)\right)^{2}} \\
& R A A E\left(t_{i}\right)=\frac{\sum_{j=1}^{n_{\text {val }}}\left|\left(y_{j}\left(t_{i}\right)-\hat{y}_{j}\left(t_{i}\right)\right)\right|}{n \times \sigma_{y\left(t_{i}\right)}}
\end{aligned}
$$

where $n_{v a l}$ is the number of validation samples, $y_{j}$ and $\hat{y}_{j}$ respectively the acceleration response result of the FEM simulation and the Kriging surrogate approximation of the validation set, and $\mu_{y_{j}}$ and $\sigma_{y_{j}}$ respectively the mean and standard deviation of the FEM simulation data. The larger the value of $R^{2}$ and the smaller the value of $R A A E$, the more accurate the meta-model.

To assess the quality of the surrogate model, the minimum of $R^{2}\left(t_{i}\right)$ and maximum of $R A A E\left(t_{i}\right)$ values over time are calculated. This is done for Kriging models with increasing Sobol set sizes. As shown in Fig. 15 and Fig. 16, when the number of Sobol samples of the training set increases from 10 to 200 , the minimum $R^{2}$ values increases from -0.15 which means low accuracy to 0.88 and the maximum $R A A E$ value decreases from 0.206 to 0.028 , which indicates that an accurate model is obtained for 200 Sobol samples. 


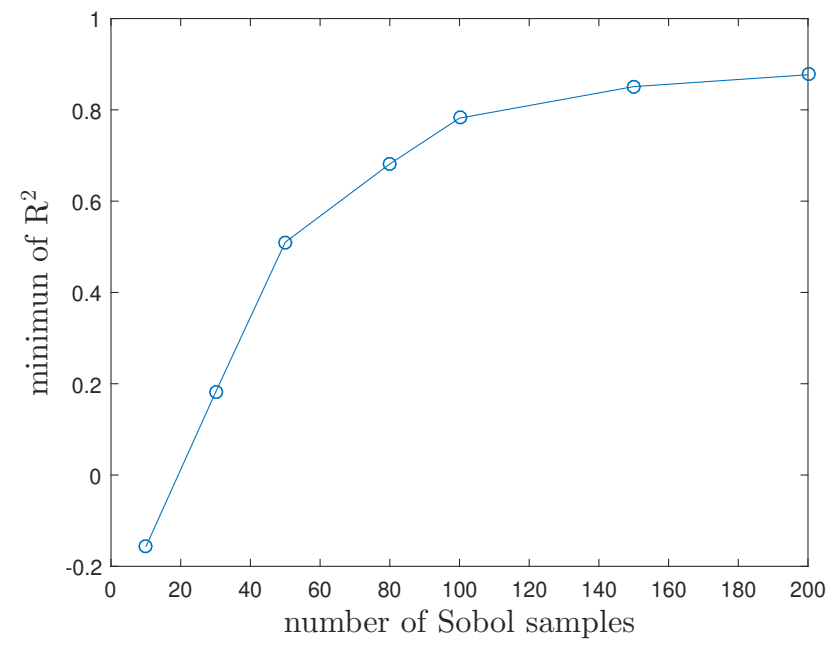

Figure 15: minimum $R^{2}$ between the Kriging and FE model predictions as a function of the number of Sobol samples

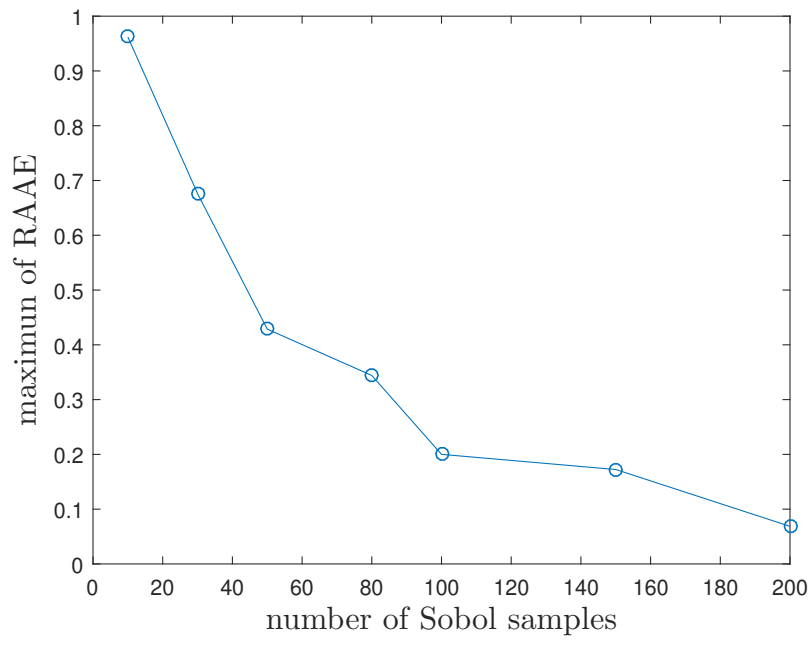

Figure 16: maximum $R A A E$ between the Kriging and FE model predictions as a function of the number of Sobol samples

In order to check the validity of the model with 200 Sobol samples for the full simulation time, $R^{2}$ and $R A A E$ are plotted as a function of $t_{i}$ in 
Fig. 17 and Fig. 18 respectively. It can be observed that both the minimum $R^{2}$ value of 0.88 and the maximum $R A A E$ value of 0.068 are reached as expected near the end of the simulation time. This is logical, as the variation in the response signal due to the uncertainty is also expected to increase over time.

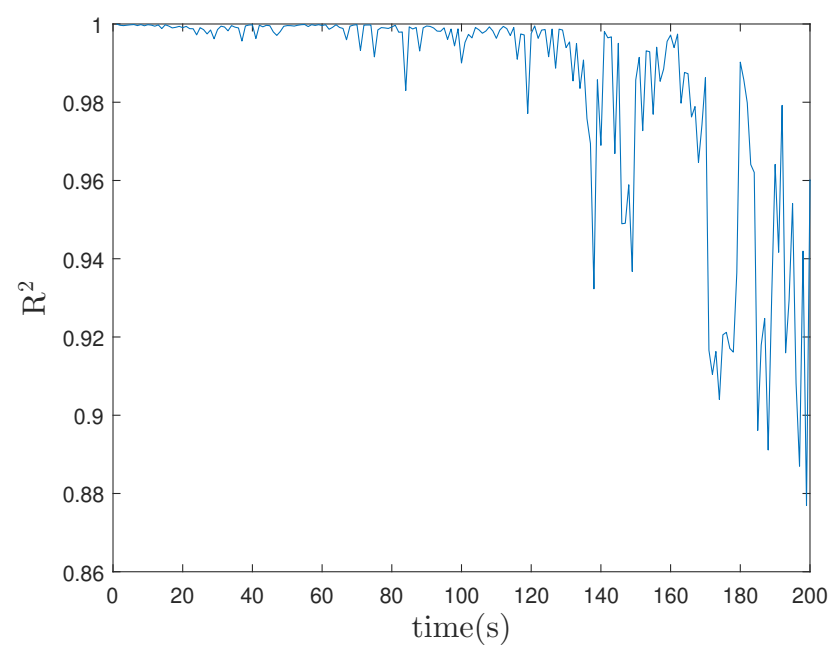

Figure 17: $R^{2}$ between the Kriging and FE model predictions as a function of simulation time

Fig. 19 finally shows the result of the propagation of the interval field for the complete landing procedure for both the acceleration and velocity response. As can be noted, the bounds on the interval field response enclose the training set perfectly.

\subsection{Comparison of interval field and random field analysis}

In this section, we compare the results of the random field and interval field propagation, both for the accuracy and computational cost. Figures 20 and 21 show the comparison of respectively the upper and lower bounds 


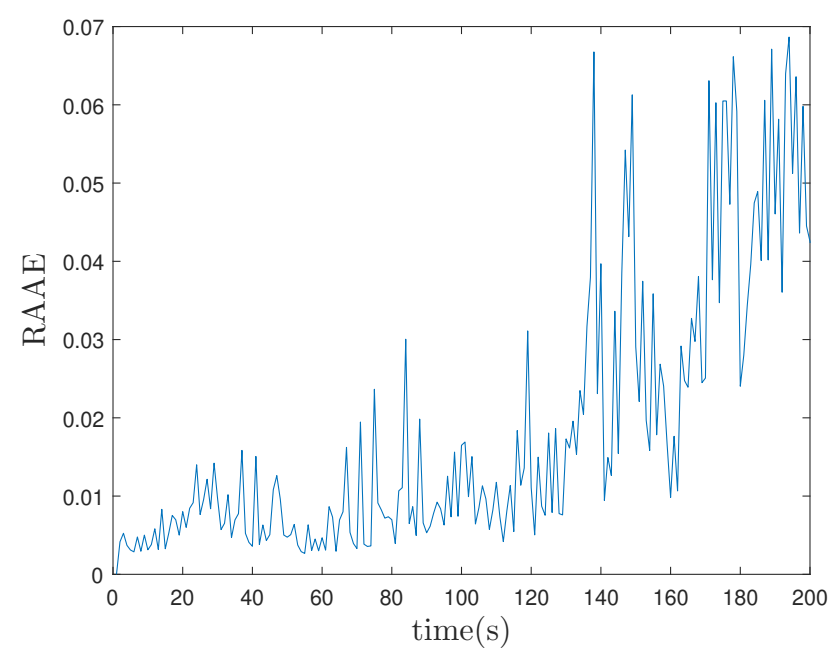

Figure 18: RAAE between the Kriging and FE model predictions as a function of simulation time

on the acceleration and velocity response of the solar wing when Young's modulus of the material is modeled via either a random or an interval field. In case of the interval field, the bounds, as obtained via Eq. 35 are shown as blue lines. In case of the random field, the $3 \sigma$ bounds of the response are illustrated as red lines. The figures also indicate the deviation between both predicted bounds. Qualitatively speaking, it can be noted that the bounds predicted as a result of both uncertain field models correspond very well to each other.

To obtain a quantitative measure of difference, the Root Mean Square Error (RMSE) values between both response bounds are calculated over the full simulation time. For the upper bound and lower bound of acceleration, the RMSE values are $198 \mathrm{~m} / \mathrm{s}^{2}$ and $183 \mathrm{~m} / \mathrm{s}^{2}$ respectively. For the upper bound and lower bound of velocity, the RMSE values are $0.26 \mathrm{~m} / \mathrm{s}$ and $0.23 \mathrm{~m} / \mathrm{s}$ respectively. This means that the results, in terms of extreme be- 


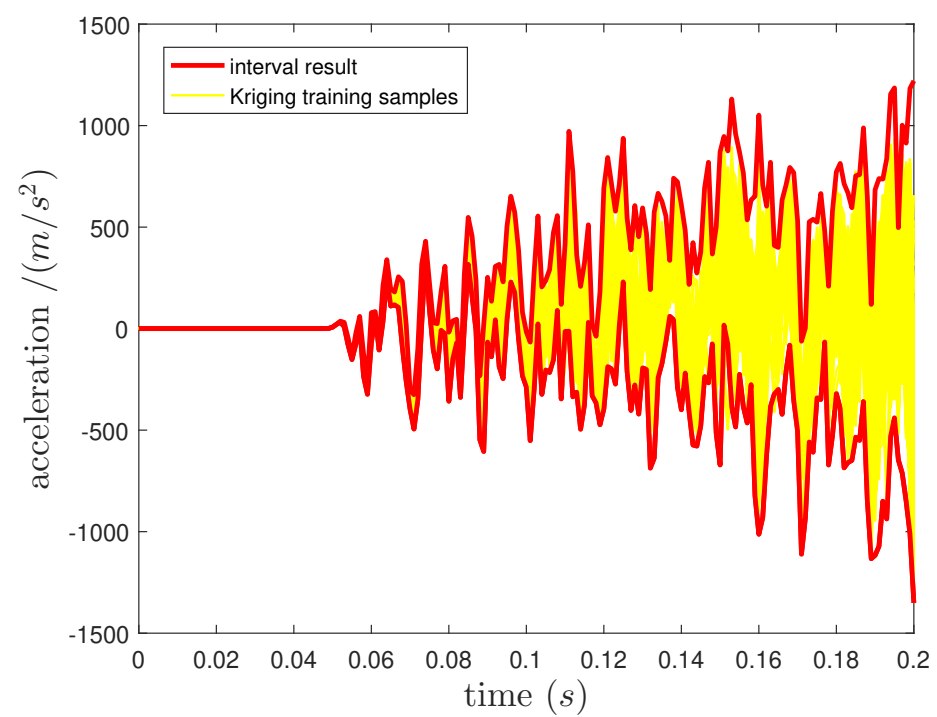

(a) interval bounds on the acceleration response compared to the 264 training samples

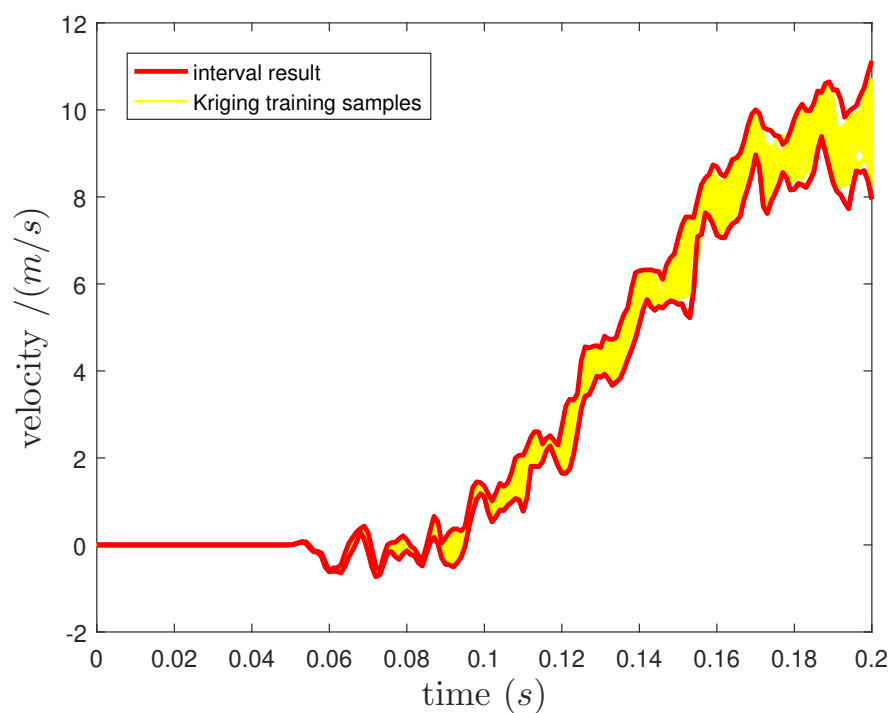

(b) interval bounds on the velocity response compared to the 264 training samples

Figure 19: Lower and upper bounds on the acceleration and velocity response of the solar wing solved using interval fields 
havior of the solar wing, predicted by propagating the interval field method and random field method correspond very well.

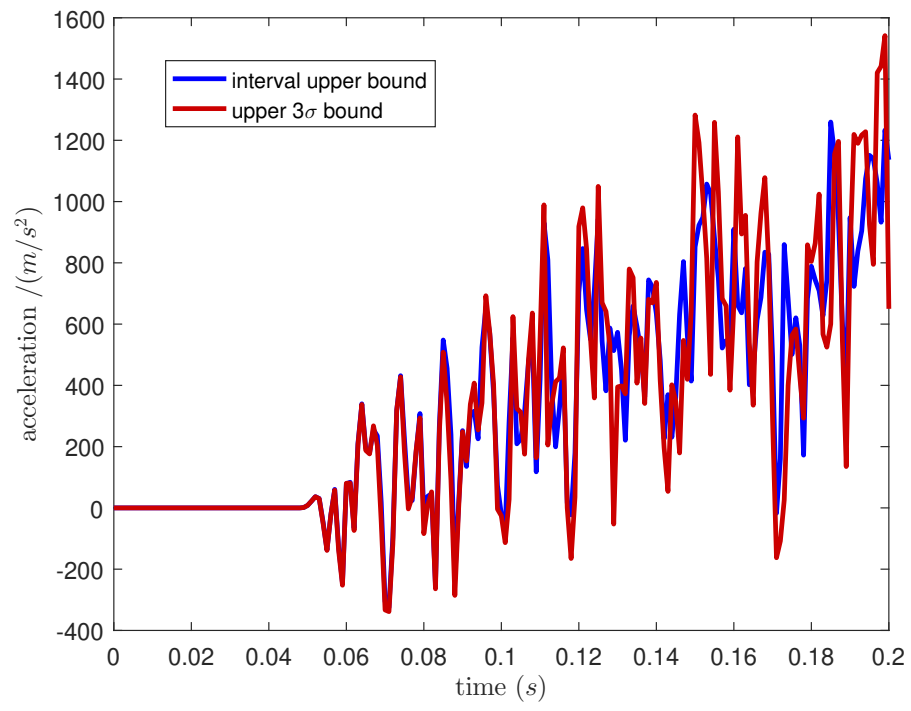

(a) comparison of upper bounds

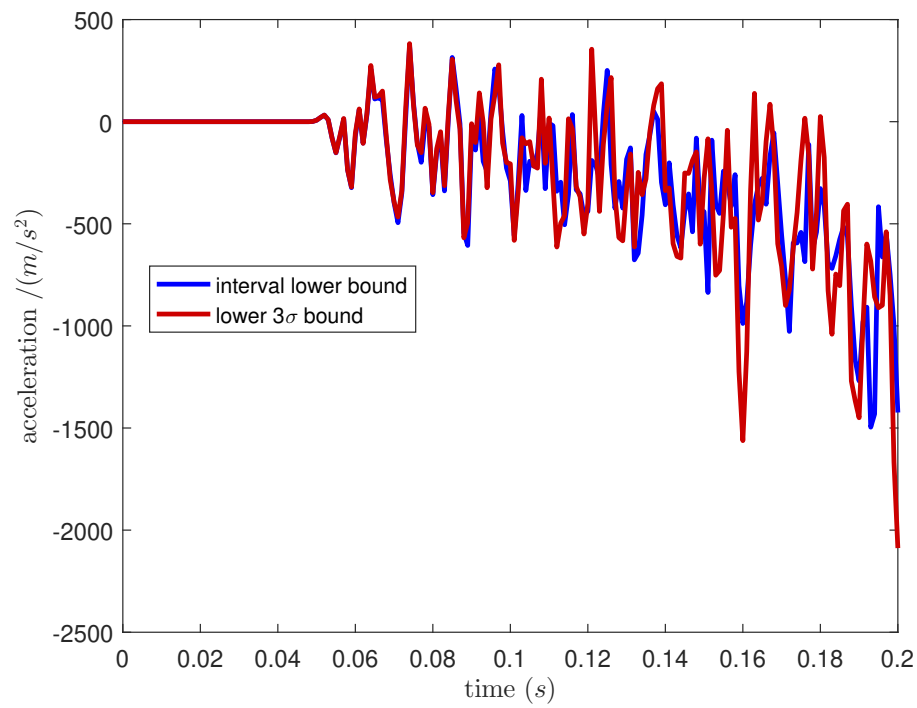

(b) comparison of lower bounds

Figure 20: Comparison of upper and lower bounds on the acceleration response resulting from interval and random field analysis 


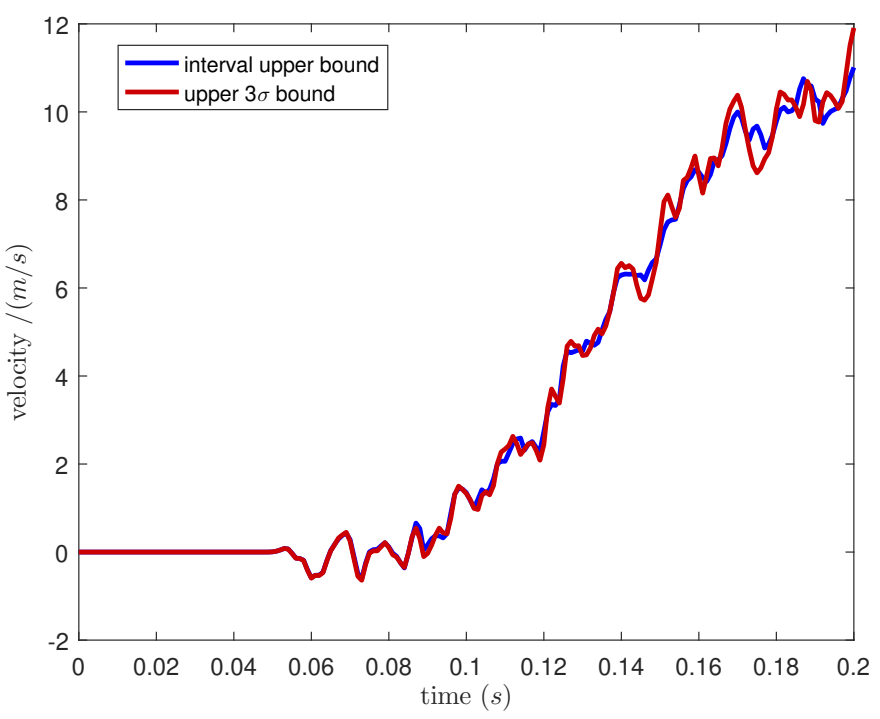

(a) comparison of upper bounds

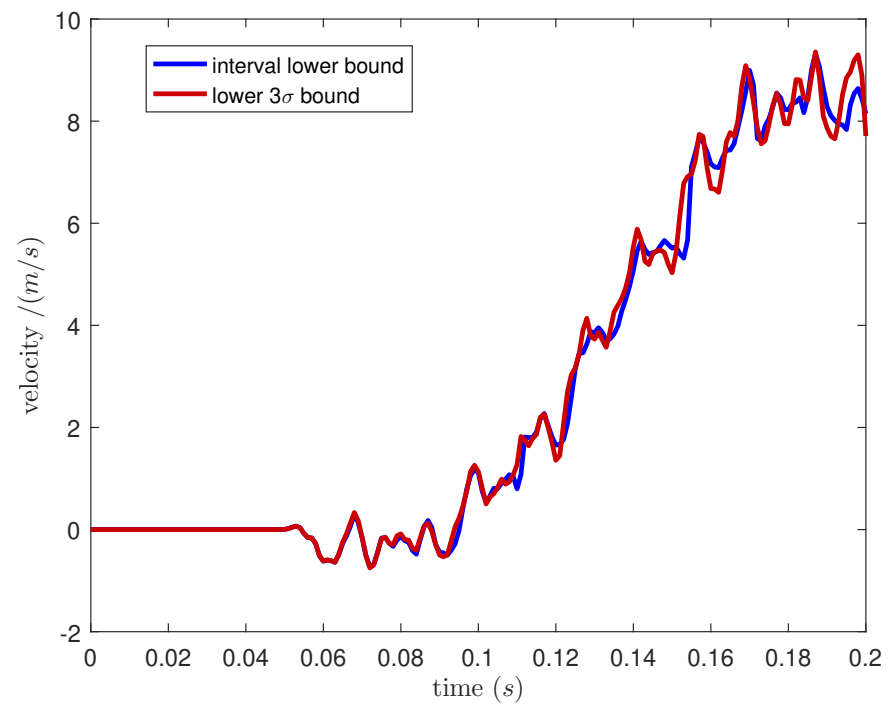

(b) comparison of lower bounds

Figure 21: Comparison of upper and lower bounds on the velocity response resulting from interval and random field analysis

To deepen the discussion, we compare the empirical cumulative distribu- 
tion function (CDF) and probability density function (PDF) obtained from propagating the random field, with the bounds obtained from the interval field at a specific time instant $(\mathrm{t}=0.08,0.12,0.16,0.2 \mathrm{~s}$ respectively) during the landing procedure. The comparison is shown in Fig. 22 and Fig. 23. The results show that the bounds predicted by propagating the interval field and the boundaries that are observed with random field method correspond very well. Furthermore, as is also clear from Fig. 22 and Fig. 23, the amount of information that is obtained by propagating a random field is higher as compared to the interval field, as also the relative likelihood of obtaining a velocity or acceleration value inside the bounds is obtained. This is for instance critical information to perform a reliability analysis.

As such, to conclude this comparison, it is shown that both the interval field and the random field give very similar results concerning the bounds on the response dynamics, even given the large difference in their respective theoretical basis. The main difference in the application of both techniques therefore lies not in the simulation results that are obtained (apart from the obvious fact that a full distribution for each time instant is obtained for the random field as opposed to only bounds in the interval case). This comparison shows that in fact, by choosing the appropriate parameters of an interval field, very similar response predictions are obtained as compared to a full random field simulation. In this study, for comparison purposes, this is a result of the tuning of the interval field towards the random field. In real life practice, it can be argued that the definition of bounds on the maximally occurring gradient in a spatial phenomenon, as required for the LIFD basis functions, are not only easier to quantify, they furthermore provide an analyst with a tool that is in some engineering applications more 


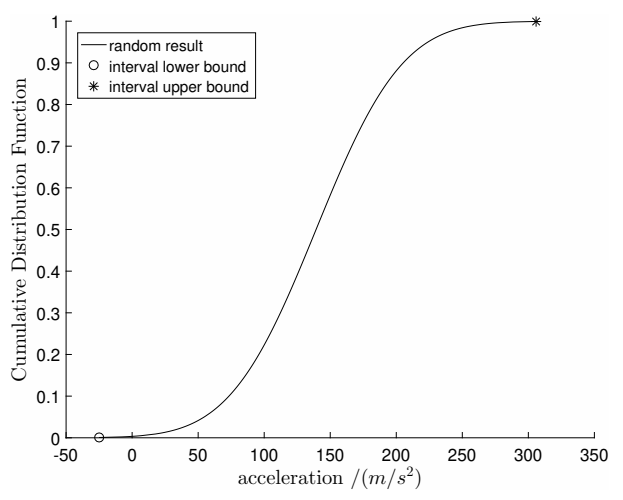

(a) $\mathrm{CDF}$ at $\mathrm{t}=0.08 \mathrm{~s}$

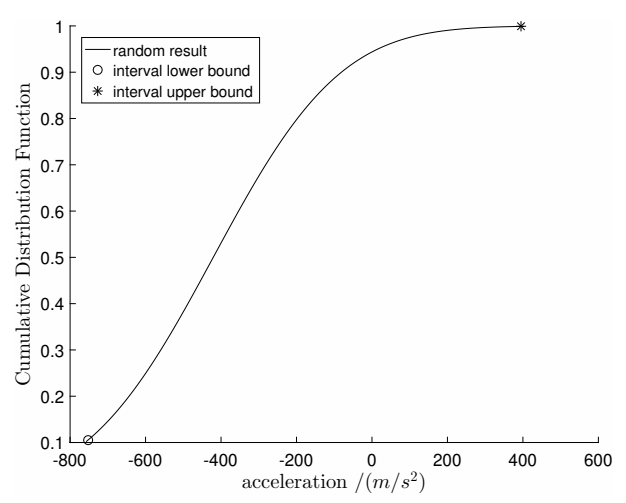

(c) $\mathrm{CDF}$ at $\mathrm{t}=0.16 \mathrm{~s}$

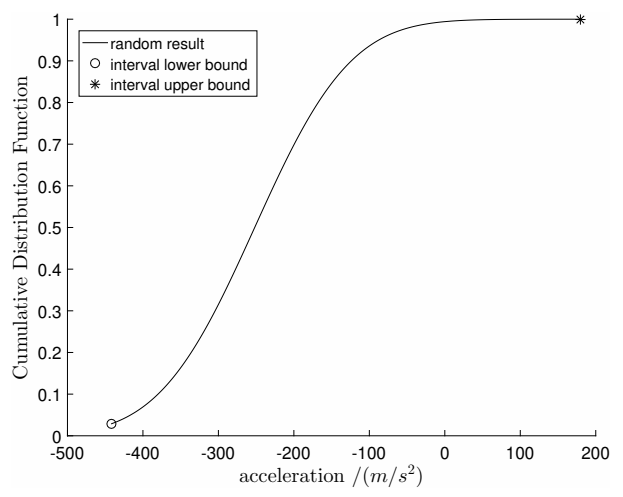

(b) $\mathrm{CDF}$ at $\mathrm{t}=0.12 \mathrm{~s}$

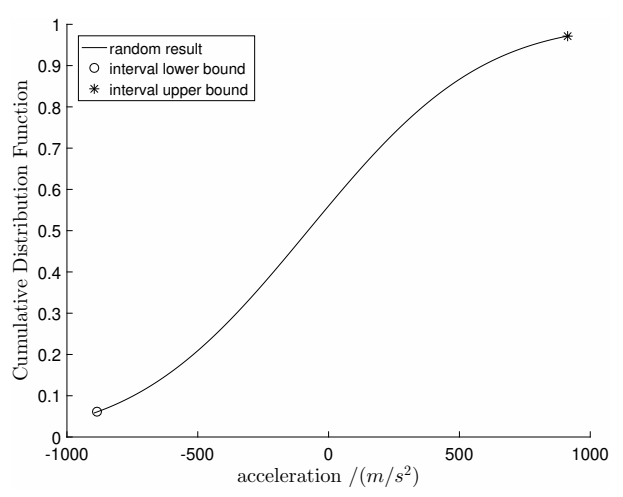

(d) $\mathrm{CDF}$ at $\mathrm{t}=0.20 \mathrm{~s}$

Figure 22: CDF obtained from the random field with the bounds obtained from the interval field

intuitive to work with as compared to the concept of a correlation length. 


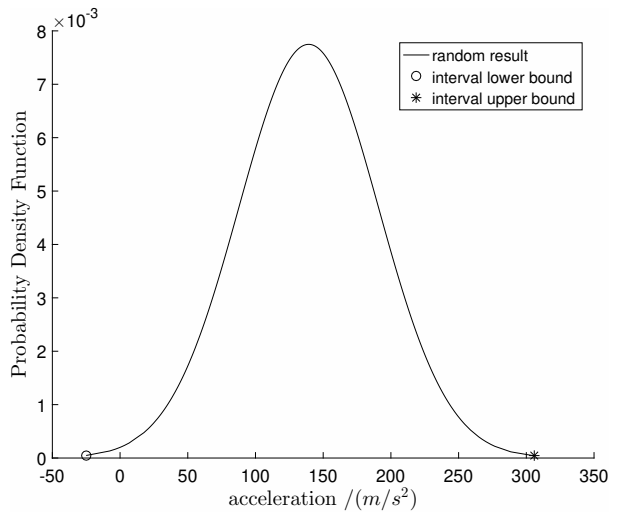

(a) PDF at $\mathrm{t}=0.08 \mathrm{~s}$

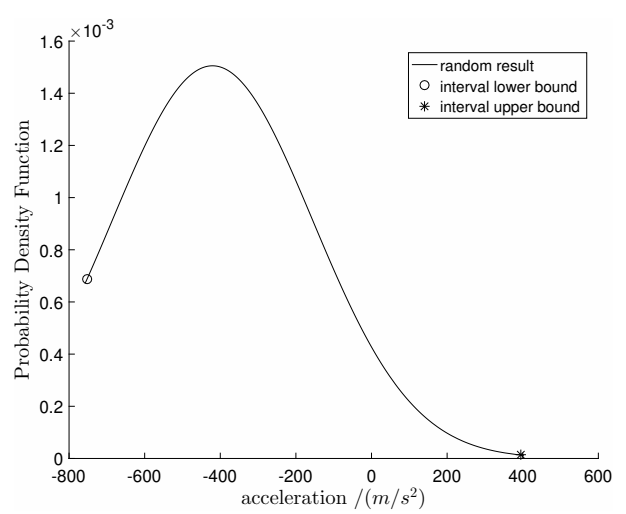

(c) PDF at $\mathrm{t}=0.16 \mathrm{~s}$

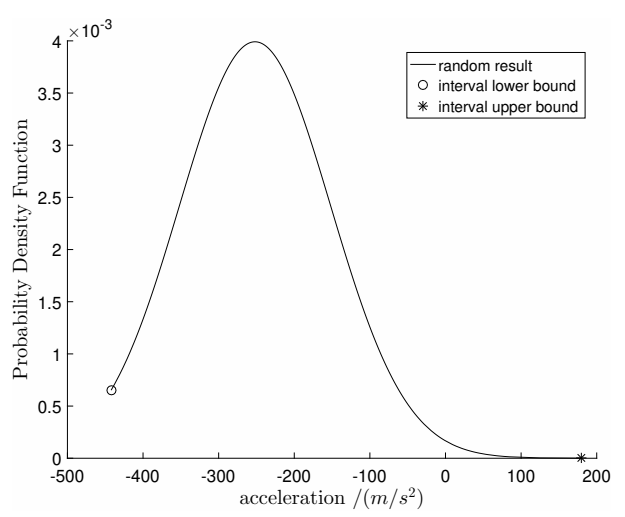

(b) PDF at $\mathrm{t}=0.12 \mathrm{~s}$

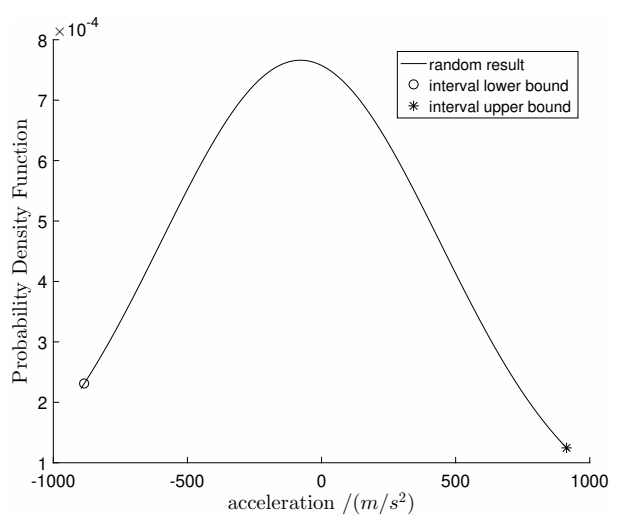

(d) $\mathrm{PDF}$ at $\mathrm{t}=0.20 \mathrm{~s}$

Figure 23: PDF obtained from the random field with the bounds obtained from the interval field 


\section{Conclusion}

The main objective of this paper is to compare two non-deterministic modeling concepts for the representation of spatial uncertainty: the wellknown framework of random fields and its more recently introduced counterpart, interval fields. Hereto, a specific case study involving the structural dynamics of a lunar lander is performed. Specifically, the effect of the spatial uncertainty that is present in Young's modulus of the carbon fiber composite solar wings on the dynamics of the lander during landing is studied. To reduce the computational cost of this lunar lander simulation, Impulse Based Substructuring is applied.

The paper presents an approach to tailor an interval field with LIFD basis functions towards a set of realizations of the spatial uncertainty, which in this case study stems from a random field model. In real life conditions, such realizations can be e.g., based on available experimental data. The case study shows that the representation of the spatial uncertainty at the input side of the model (i.e., Young's modulus of the solar wing) is highly comparable between the random field and interval field model when the latter is tailored towards the former. Further, when propagated towards the dynamic response of the model, also highly comparable results are obtained between both modeling approaches.

The main difference however between both approaches lies in the requirements for the definition of the uncertainty model. Random fields on the one hand require the definition of a full auto-correlation structure to enable the generation of realizations via the Karhunen-Loeève expansion. In practice, this usually boils down to the selection of an appropriate probability density function, auto-correlation function and corresponding cor- 
relation length parameter. The interval field based on LIFD on the other hand only requires the definition of the bounds on the quantity of interest and the maximum gradient the realizations should abide by. It is clear the requirements in terms of data for the latter are far less stringent than for the former. As such, this paper shows that, by placing less stringent needs on the requirement of data for the estimation of spatial uncertainty, comparable results in terms of bounds on the response are obtained by using an interval field as compared to a random field.

\section{Acknowledgment}

The authors acknowledge the support for the work presented in this paper from the China Scholarship Council, Beijing Institute of Technology and KU Leuven Internal Funds. The Research Foundation Flanders is gratefully acknowledged for the support of Matthias Faes under grant number 12P3519N, and of Maurice Imholz under grant G0C2218N.

\section{References}

[1] M. Faes, D. Moens, Recent trends in the modeling and quantification of nonprobabilistic uncertainty, Archives of Computational Methods in Engineering (2019) $1-39$.

[2] G. Stefanou, The stochastic finite element method: past, present and future, Computer methods in applied mechanics and engineering 198 (9-12) (2009) 1031-1051.

[3] M. Beer, S. Ferson, V. Kreinovich, Imprecise probabilities in engineering analyses, Mechanical systems and signal processing 37 (1-2) (2013) 4-29.

[4] H. Agarwal, J. E. Renaud, E. L. Preston, D. Padmanabhan, Uncertainty quantification using evidence theory in multidisciplinary design optimization, Reliability Engineering \& System Safety 85 (1-3) (2004) 281-294. 
[5] D. Moens, M. Hanss, Non-probabilistic finite element analysis for parametric uncertainty treatment in applied mechanics: Recent advances, Finite Elements in Analysis and Design 47 (1) (2011) 4-16.

[6] M. Faes, M. Broggi, E. Patelli, Y. Govers, J. Mottershead, M. Beer, D. Moens, A multivariate interval approach for inverse uncertainty quantification with limited experimental data, Mechanical Systems and Signal Processing 118 (2019) 534-548.

[7] M. Faes, D. Moens, Imprecise random field analysis with parametrized kernel functions, Mechanical Systems and Signal Processing 134 (2019) 106334.

[8] H. Ahmadian, J. Mottershead, M. Friswell, Regularisation methods for finite element model updating, Mechanical Systems and Signal Processing 12 (1) (1998) $47-64$.

[9] A. Balu, B. Rao, High dimensional model representation based formulations for fuzzy finite element analysis of structures, Finite Elements in Analysis and Design 50 (2012) 217-230.

[10] C. B. Barber, D. P. Dobkin, D. P. Dobkin, H. Huhdanpaa, The quickhull algorithm for convex hulls, ACM Transactions on Mathematical Software (TOMS) 22 (4) (1996) 469-483.

[11] J. L. Beck, S.-K. Au, Bayesian updating of structural models and reliability using markov chain monte carlo simulation, Journal of engineering mechanics 128 (4) (2002) 380-391.

[12] J. L. Beck, L. S. Katafygiotis, Updating models and their uncertainties. i: Bayesian statistical framework, Journal of Engineering Mechanics 124 (4) (1998) 455-461.

[13] W. Betz, I. Papaioannou, D. Straub, Numerical methods for the discretization of random fields by means of the Karhunen-Loève expansion, Computer Methods in Applied Mechanics and Engineering 271 (2014) 109-129. doi:10.1016/j.cma.2013.12.010.

URL http://dx.doi.org/10.1016/j.cma.2013.12.010

[14] D. Moens, M. De Munck, W. Desmet, D. Vandepitte, Numerical dynamic analysis of uncertain mechanical structures based on interval fields, in: IUTAM symposium on the vibration analysis of structures with uncertainties, Springer, 2011, pp. 71-83.

[15] W. Verhaeghe, W. Desmet, D. Vandepitte, D. Moens, Interval fields to represent 
uncertainty on the output side of a static fe analysis, Computer Methods in Applied Mechanics and Engineering 260 (2013) 50-62.

[16] G. Muscolino, A. Sofi, Bounds for the stationary stochastic response of truss structures with uncertain-but-bounded parameters, Mechanical Systems and Signal Processing 37 (1-2) (2013) 163-181.

[17] D. Wu, W. Gao, Uncertain static plane stress analysis with interval fields, International Journal for Numerical Methods in Engineering 110 (13) (2017) 1272-1300.

[18] A. Sofi, Structural response variability under spatially dependent uncertainty: stochastic versus interval model, Probabilistic Engineering Mechanics 42 (2015) 78 86.

[19] S. Yin, D. Yu, H. Yin, B. Xia, Interval and random analysis for structure-acoustic systems with large uncertain-but-bounded parameters, Computer Methods in Applied Mechanics and Engineering 305 (2016) 910-935.

[20] W. Gao, D. Wu, K. Gao, X. Chen, F. Tin-Loi, Structural reliability analysis with imprecise random and interval fields, Applied Mathematical Modelling 55 (2018) 49-67.

[21] D. Wu, W. Gao, Hybrid uncertain static analysis with random and interval fields, Computer Methods in Applied Mechanics and Engineering 315 (2017) 222-246.

[22] J. Feng, D. Wu, W. Gao, G. Li, Hybrid uncertain natural frequency analysis for structures with random and interval fields, Computer Methods in Applied Mechanics and Engineering 328 (2018) 365-389.

[23] M. Faes, G. D. Sabyasachi, D. Moens, Hybrid spatial uncertainty analysis for the estimation of imprecise failure probabilities in laser sintered pa-12 parts, Computers \& Mathematics with Applications 78 (7) (2019) 2395-2406.

[24] S. Chen, L. Li, S. Zhou, Z. Chen, Z. Yue, Multi-domain substructures synthesis with general joints for the dynamics of large structures, Aip Advances 7 (10) (2017) 105007.

[25] P. D. Spanos, R. Ghanem, Stochastic finite element expansion for random media, Journal of engineering mechanics 115 (5) (1989) 1035-1053.

[26] K. Phoon, S. Huang, S. Quek, Simulation of second-order processes using karhunenloeve expansion, Computers \& structures 80 (12) (2002) 1049-1060. 
[27] K. Phoon, H. Huang, S. Quek, Simulation of strongly non-gaussian processes using karhunen-loeve expansion, Probabilistic engineering mechanics 20 (2) (2005) 188198.

[28] R. Ghanem, Ingredients for a general purpose stochastic finite elements implementation, Computer Methods in Applied Mechanics and Engineering 168 (1-4) (1999) $19-34$.

[29] H. G. Matthies, A. Keese, Galerkin methods for linear and nonlinear elliptic stochastic partial differential equations, Computer methods in applied mechanics and engineering 194 (12-16) (2005) 1295-1331.

[30] M. Grigoriu, Simulation of stationary non-gaussian translation processes, Journal of engineering mechanics 124 (2) (1998) 121-126.

[31] R. G. Ghanem, P. D. Spanos, Stochastic finite elements: a spectral approach, Courier Corporation, 2003.

[32] C. G. Bucher, Adaptive sampling - an iterative fast monte carlo procedure, Structural safety 5 (2) (1988) 119-126.

[33] R. Melchers, Importance sampling in structural systems, Structural safety 6 (1) (1989) 3-10.

[34] S.-K. Au, J. L. Beck, Estimation of small failure probabilities in high dimensions by subset simulation, Probabilistic Engineering Mechanics 16 (4) (2001) 263-277. doi:https://doi.org/10.1016/S0266-8920(01)00019-4.

URL http://www.sciencedirect.com/science/article/pii/S0266892001000194

[35] M. Faes, J. Sadeghi, M. Broggi, M. de Angelis, E. Patelli, M. Beer, D. Moens, On the Robust Estimation of Small Failure Probabilities for Strong Nonlinear Models, ASCE-ASME J Risk and Uncert in Engrg Sys Part B Mech Engrg 5 (4), 041007 (09 2019). arXiv:https://asmedigitalcollection.asme.org/risk/articlepdf/5/4/041007/5873805/risk_005_04_041007.pdf, doi:10.1115/1.4044044.

URL https://doi.org/10.1115/1.4044044

[36] M. Imholz, D. Vandepitte, D. Moens, Application of interval fields to fit experimental data on deepdrawn components, in: Proceedings of the joint ICVRAM ISUMA UNCERTAINTIES conference (2), 2018.

[37] M. Faes, J. Cerneels, D. Vandepitte, D. Moens, Identification and quantification 
of multivariate interval uncertainty in finite element models, Computer Methods in applied mechanics and engineering 315 (2017) 896-920.

[38] M. Faes, Interval methods for the identification and quantification of inhomogeneous uncertainty in finite element models, Ph.D. thesis, Ph. D. thesis, KU Leuven, Department of Mechanical Engineering (2017).

[39] M. Faes, J. Cerneels, D. Vandepitte, D. Moens, Identification of interval fields for spatial uncertainty representation in finite element models, in: Proceedings of the ECCOMAS Congress, Vol. 30, 2016, pp. 27-30.

[40] M. Imholz, D. Vandepitte, D. Moens, Derivation of an input interval field decomposition based on expert knowledge using locally defined basis functions, in: UNCECOMP 2015-1st ECCOMAS Thematic Conference on Uncertainty Quantification in Computational Sciences and Engineering, 2015, pp. 529-547.

[41] M. Imholz, D. Vandepitte, D. Moens, Analysis of the effect of uncertain clamping stiffness on the dynamical behaviour of structures using interval field methods, in: Applied mechanics and materials, Vol. 807, Trans Tech Publ, 2015, pp. 195-204.

[42] C. De Boor, A. Ron, On multivariate polynomial interpolation, Constructive Approximation 6 (3) (1990) 287-302.

[43] D. McDonald, W. Grantham, W. Tabor, M. Murphy, Response surface model development for global/local optimization using radial basis functions, in: 8th Symposium on Multidisciplinary Analysis and Optimization, 2000, p. 4776.

[44] Z. Deng, Z. Guo, X. Zhang, Interval model updating using perturbation method and radial basis function neural networks, Mechanical Systems and Signal Processing 84 (2017) 699-716.

[45] E. Patelli, Y. Govers, M. Broggi, H. M. Gomes, M. Link, J. E. Mottershead, Sensitivity or bayesian model updating: a comparison of techniques using the dlr airmod test data, Archive of Applied Mechanics 87 (5) (2017) 905-925.

[46] J. D. Martin, T. W. Simpson, Use of kriging models to approximate deterministic computer models, AIAA journal 43 (4) (2005) 853-863.

[47] H. H. Khodaparast, Y. Govers, S. Adhikari, M. Link, M. Friswell, J. Mottershead, J. Sienz, Fuzzy model updating and its application to the dlr airmod test structure, in: Proceeding of ISMA, 2014, pp. 3109-3117. 
[48] Y. Yu, D. Wu, W. Gao, Stochastic chemo-physical-mechanical degradation analysis on hydrated cement under acidic environments, Applied Mathematical Modelling 78 (2020) $75-97$.

[49] M. Faes, D. Moens, Multivariate dependent interval finite element analysis via convex hull pair constructions and the extended transformation method, Computer Methods in Applied Mechanics and Engineering 347 (2019) 85-102.

[50] R. Jin, W. Chen, T. W. Simpson, Comparative studies of metamodelling techniques under multiple modelling criteria, Structural and multidisciplinary optimization 23 (1) (2001) 1-13. 